\title{
WEAK FACTORIZATIONS OF OPERATORS IN THE GROUP VON NEUMANN ALGEBRAS OF CERTAIN AMENABLE GROUPS AND APPLICATIONS
}

\author{
M. FILALI, M. NEUFANG, AND M. SANGANI MONFARED
}

\begin{abstract}
Let $G$ be a compact group whose local weight $b(G)$ has uncountable cofinality. Let $H$ be an amenable locally compact group and $A(G \times H)$ be the Fourier algebra of $G \times H$. We prove that the group von Neumann algebra $V N(G \times H)=A(G \times H)^{*}$ has the weak uniform $A(G \times H)^{* *}$ factorization property of level $b(G)$. As a corollary we show that $A(G \times H)$ is strongly Arens irregular, and the topological centre of $U C_{2}(G \times H)^{*}$ is equal to the Fourier-Stieltjes algebra $B(G \times H)$.
\end{abstract}

\section{INTRODUCTION}

Let $A$ be a Banach algebra and $\kappa$ be a cardinal number. Then $A^{*}$ is said to have the left $A^{* *}$ factorization property of level $\kappa$ if for every bounded family of functionals $\left(h_{\alpha}\right)_{\alpha \in I} \subset A^{*}$ with $|I|=\kappa$, there exist a bounded family $\left(\psi_{\alpha}\right)_{\alpha \in I}$ in $A^{* *}$ and a single functional $h \in A^{*}$ such that the factorization formula $h_{\alpha}=\psi_{\alpha} \cdot h$ holds for all $\alpha \in I$. The first such factorization result was obtained by the second named author in [43] for $L^{\infty}(G)=L^{1}(G)^{*}$, where $G$ is a locally compact non-compact group, and $\kappa$ is the compact covering number of $G$. The existence of such factorizations can be used in the study of topological centres of the biduals of Banach algebras, as shown by Neufang [44, 45].

In [1] Arens showed that the product on a Banach algebra $A$ can be extended in two different ways to its double dual $A^{* *}$. When the two extended products are identical, $A$ is called Arens regular. This is the case, for example, if $A$ is a $C^{*}$-algebra (or, more generally, an operator algebra). The left (respectively, right) topological centre of $A^{* *}$ is the subspace consisting of all $m$ in $A^{* *}$ such that left (respectively, right) multiplication by $m$ with respect to either product is the same. If the left (respectively, right) topological centre of $A^{* *}$ is equal to $A$, then, following Dales-Lau [5], $A$ is called left (respectively, right) strongly Arens irregular; if $A$ is both left and right strongly Arens irregular, it is called strongly Arens irregular. In the case that $A$ is commutative,

2000 Mathematics Subject Classification. 22D15, 43A20, 43A30, 43A40, 43A75.

Key words and phrases. Amenable groups, group von Neumann algebra, factorization, Fourier algebra, topological centre, unitary representations, ideals, cancellable sets. 
both topological centres coincide with the algebraic centre of $A^{* *}$ (endowed with either Arens product). A complete characterization of the topological centres is often difficult. For the case of the group algebra $L^{1}(G)$, following earlier works by Civin-Yood [3], Young [50], and Işik-Pym-Ülger [29], finally in 1988, Lau and Losert [36] showed that $L^{1}(G)$ is strongly Arens irregular for all locally compact groups. More information about the results on topological centres of Banach algebras can be found in [14]. For another new approach leading to the determination of topological centres including those of $L^{1}(G)^{* *}, L U C(G)^{*}$ and their corresponding weighted algebras, see Filali-Salmi $[12,13])$.

If $\lambda$ is the left regular representation of $G$ on $L^{2}(G)$ defined by $\lambda(t) f(x)=f\left(t^{-1} x\right)$, then the Fourier algebra $A(G)$ is the collection of all functions $u(x)=(\lambda(x) f \mid g)=\bar{g} * \check{f}(x)(x \in G)$, where $f, g \in L^{2}(G)$. The group von Neumann algebra $V N(G) \cong A(G)^{*}$ is the closure in the weak operator topology of the linear span of $\{\lambda(x): x \in G\}$ in $\mathcal{B}\left(L^{2}(G)\right.$ ) (see Eymard [8]). In harmonic analysis, $A(G)$ is considered the dual object of the group algebra $L^{1}(G)$. Indeed, when $G$ is abelian, $A(G) \cong L^{1}(\widehat{G})$ via the Fourier transformation. The analogues of many results on $L^{1}(G)$ have been proved for $A(G)$, which have helped to better understand the nature of the duality between $L^{1}(G)$ and $A(G)$. However, unlike the case of $L^{1}(G)$, the characterization of the topological centre of the bidual of the (commutative) Banach algebra $A(G)$ has resisted a solution for many years, and is still not complete.

In this paper we show that for any compact group $G$ whose local weight has uncountable cofinality, and any amenable group $H$, the algebra $A(G \times H)$ is strongly Arens irregular (Corollary 4.7). As an implication of our result, we mention the following interesting phenomenon: while by a result of Losert [42], $A(S U(3))$ is not strongly Arens irregular, the Fourier algebra of a suitable power of $S U(3)$ - for instance $S U(3)^{\aleph_{1}}$ - is in fact strongly Arens irregular (cf. [38] for the case of the power of size $\aleph_{0}$ ). The proof of our result relies on the existence of a 'weak' factorization property for the group von Neumann algebra $V N(G \times H)$ which we shall prove in Theorem 4.6. In obtaining our factorization formulae (23) and (36) we have been influenced by the role of right cancellable points in the study of topological centres of Banach algebras and semigroup compactifications as shown in Filali-Pym [10] and Filali-Salmi $[11,12,13]$. In fact, as in the case of right cancellable points discussed in the above papers, we obtain the terms $C_{k l}^{\lambda}$ and $\widetilde{\xi}_{k l}^{\lambda}$ in our factorization formulae from some cluster points of a 'thin set'. In our case, the thin set consists of coordinate functions of carefully selected irreducible unitary representations of $G$.

To put our result on the (topological) centre of $A(G)^{* *}$ in a proper context, we briefly survey some of the earlier results on the subject. In 1989, Lau and Wong [39] showed that if $G$ is amenable, then $A(G)$ is Arens regular if and only if $G$ is finite. Later, Forrest [16] obtained 
an analogous result for large classes of discrete groups. In addition, he also showed that if $G$ is a locally compact group for which $A(G)$ is Arens regular and if $H$ is an amenable subgroup of $G$, then $H$ is finite. As observed by Forrest, from this result it follows immediately that $A(G)$ is not Arens regular if $G$ is the Ol'shanskii's famous example of a discrete non-amenable group for which every non-trivial proper subgroup is infinite [46]. In [37, 38] Lau and Losert showed that $A(G)$ is strongly Arens irregular for large classes of amenable groups which includes discrete amenable groups, second countable amenable groups $G$ such that $\overline{[G, G]}$ is not open in $G$, and countably infinite product of second countable locally compact groups $G_{i}, i=0,1,2, \ldots$ where each $G_{i}$ is a non-trivial compact group for $i>0$. In his survey paper [35] on how the structures of $A(G)$ and the Fourier-Stieltjes algebra $B(G)$ relate to the amenability of $G$, Lau posed a series of open interesting problems including that of the determination of the centre of $A(G)^{* *}$. Ten years later, the same question was again asked by Kaniuth and Lau in their survey article [31], where they presented developments on how amenability of the Fourier algebra $A(G)$ and the Fourier-Stieltjes algebra $B(G)$ can be characterized with properties of subalgebras of $A(G)$ and $B(G)$ and their duals. Recently, Losert [40] has proved that if $G$ is a discrete group containing $\mathbb{F}_{r}$ (the free group with $r$ generators, where $r \geq 2$ is finite) then $A(G)$ is not strongly Arens irregular. In 2008, Losert announced in his lectures [42] that $A(G)$ is not strongly Arens irregular when $G$ is either the compact group $S U(3)$ or the locally compact group $S L(2, \mathbb{R})$, but $A(G)$ is strongly Arens irregular when $G$ is the compact group $S U(2)$.

We should also note that the study of topological centres is related to many other interesting problems arising in abstract harmonic analysis. For instance, if $M_{c b} A(G)$ is the space of completely bounded multipliers of $A(G)$ (or Herz-Schur multipliers) and $M_{b} A(G)$ is the space of bounded multipliers of $A(G)$, then $B(G) \subseteq M_{c b} A(G) \subseteq M_{b} A(G)$ for any locally compact group, and, as is well known, these spaces are equal isometrically when $G$ is amenable. In [41], it was shown that $B(G) \neq M_{b} A(G)$ whenever $G$ is not amenable. Also, in [2], it was shown that $M_{c b} A(G) \neq M_{b} A(G)$ when $G$ is a noncommutative free group. It was open for quite a long time whether the latter inclusion is strict when $G$ is not amenable. See for example [6], [47], [32] and [48] for more details and references related to this problem. However, the recent techniques leading Losert to the (topological) centre of $A(G)^{* *}$ when $G=S L(2, \mathbb{R})$ enables him also to show that $M_{c b} A(G)=M_{b} A(G)$ in this case [42].

Despite the above progress, it is still not known exactly when $A(G)$ is strongly Arens irregular. For further results on the centre of $A(G)^{* *}$ see $[18,19,25,26,27,28]$. 
In Section 2 we briefly discuss the preliminaries and the notation used in this paper.

The main result in Section 3 is Theorem 3.11 in which we prove that if $G$ is a compact group whose local weight $b(G)$ has uncountable cofinality, then $V N(G)$ has the left weak uniform $A(G)^{* *}$ factorization property of level $b(G)$. In Corollary 3.14 we show that for these groups, the centre of $A(G)^{* *}$ is exactly $A(G)$ (in other words, $A(G)$ is strongly Arens irregular), and that every left $A(G)^{* *}$ module homomorphism of $A(G)^{*}$ is automatically bounded and normal (that is, $w^{*}-w^{*}$-continuous).

In Section 4, we extend our results in Section 3 to the product group $G \times H$ where $G$ is as above, and $H$ is an arbitrary amenable locally compact group.

We would like to thank the referee for the valuable comments and corrections. A large part of this work was done when the first author was visiting Universities of Carleton and Windsor in September 2007 and March 2008. He would like to express his warm thanks for the kind hospitality and support.

\section{Preliminaries and Notation}

For a set $S$ we write $|S|$ to denote the cardinality of $S$. An ordinal number $\alpha$ is called an initial ordinal if for each ordinal $\beta$ such that $|\alpha|=|\beta|$, we have $\alpha \leq \beta$ (equivalently, if $\beta<\alpha$ implies that $|\beta|<|\alpha|$ ). Let $\alpha>0$ be a limit ordinal (that is, $\alpha$ is not a successor ordinal), a subset $A \subset \alpha$ is called cofinal in $\alpha$ if $\sup A=\alpha$. The cofinality of $\alpha$ is, by definition, the least limit ordinal $\beta$ such that there is an increasing $\beta$-sequence $\left(\alpha_{\xi}\right)_{\xi<\beta}$ with $\lim _{\xi \rightarrow \beta} \alpha_{\xi}=\alpha$ (cf. [30]). Let $G$ be a locally compact group. We denote by $b(G)$ the local weight of $G$, i.e., the smallest cardinality of an open base at the identity element $e$ of $G$. If $G$ is non-metrizable, then $b(G)>\aleph_{0}$ by Hewitt-Ross [20, Theorem 8.7].

Let $f$ be a function on $G$ and $t \in G$. Then the left and right translations of $f$ by $t$ are denoted by ${ }_{t} f$ and $f_{t}$ and are defined by ${ }_{t} f(x)=f(t x)$ and $f_{t}(x)=f(x t)(x \in G)$. The function $\check{f}$ on $G$ is defined by $\check{f}(x)=f\left(x^{-1}\right)$.

Let $V$ be a (continuous) unitary representation of $G$ on a Hilbert space $H$. If $\left(e_{i}\right)_{i \in I}$ is an orthonormal basis of $H$, then the continuous functions $v_{i j}(x)=\left(V(x) e_{j} \mid e_{i}\right)(x \in G)$ are called the coordinate functions of $V$ with respect to $\left(e_{i}\right)_{i \in I}$. We denote the conjugate (contragredient) representation of $V$ by $\bar{V}$. If $V=\left(v_{i j}\right)$ in an orthonormal basis of $H$, then we have $\bar{V}=\left(\bar{v}_{i j}\right)$ in the same basis. If $V_{1}$ and $V_{2}$ are two unitary representations of $G$, we denote by $V_{1} \otimes V_{2}$ the tensor product of $V_{1}$ and $V_{2}$ defined by $V_{1} \otimes V_{2}(x)=V_{1}(x) \otimes V_{2}(x)$ $(x \in G)$. Note that $V_{1} \otimes V_{2}$ is a unitary representation of $G$, but in general $V_{1} \otimes V_{2}$ is not irreducible, even if $V_{1}$ and $V_{2}$ are. Let $G_{0}$ be 
a closed subgroup of $G$, and let $V_{0}$ be a unitary representation of $G_{0}$ on a Hilbert space $H_{0}$. We say that $V$ is a unitary extension of $V_{0}$ if $H_{0}$ is a closed subspace of $H$, and $V(x) \xi=V_{0}(x) \xi$ for all $x \in G_{0}$ and $\xi \in H_{0}$. If $G$ is a compact group, then any unitary representation $V_{0}$ of $G_{0}$ has a unitary extension to $G$; moreover, the extension can be chosen to be irreducible if $V_{0}$ is irreducible (cf. [21, Theorem 27.46]). Let $V_{\gamma}: G \longrightarrow \mathcal{B}\left(H_{\gamma}\right)(\gamma \in \Gamma)$ be a family of unitary representations of $G$, and let $V=\oplus_{\gamma} V_{\gamma}: G \longrightarrow \mathcal{B}\left(\oplus_{\gamma} H_{\gamma}\right)$ be their direct sum. We say that a unitary representation $W$ of $G$ is contained in $V$ (or is a summand of $V$ ) if $W$ is equivalent to $V_{\gamma}$ for some $\gamma \in \Gamma$. We let $\widehat{G}$ be the set of all equivalent classes of irreducible unitary representations of $G$.

Let $A$ be a Banach algebra and $E$ be a Banach $A$-bimodule. Then $E^{*}$ is a Banach $A$-bimodule by the module actions defined by

$$
\langle a \cdot f, x\rangle=\langle f, x \cdot a\rangle, \quad\langle f \cdot a, x\rangle=\langle f, a \cdot x\rangle \quad\left(a \in A, x \in E, f \in E^{*}\right) .
$$

In particular both $A^{*}$ and $A^{* *}$ are Banach $A$-bimodules. The space $A^{* *}$ is a Banach algebra under both the first $\square$ and the second $\diamond$ Arens products defined by $\left(a \in A, f \in A^{*}, \Phi, \Psi \in A^{* *}\right)$ :

$$
\begin{aligned}
& \langle\Phi \square \Psi, f\rangle=\langle\Phi, \Psi \cdot f\rangle, \quad\langle\Phi \diamond \Psi, f\rangle=\langle\Psi, f \cdot \Phi\rangle, \\
& \langle\Psi \cdot f, a\rangle=\langle\Psi, f \cdot a\rangle, \quad\langle f \cdot \Phi, a\rangle=\langle\Phi, a \cdot f\rangle .
\end{aligned}
$$

A Banach algebra $A$ is called Arens regular if $\square$ and $\diamond$ coincide on $A^{* *}$. The left topological centre of $A^{* *}$ is defined by

$$
\mathfrak{Z}_{t}^{(l)}\left(A^{* *}\right)=\left\{\Phi \in A^{* *}: \Phi \square \Psi=\Phi \diamond \Psi \text { for all } \Psi \in A^{* *}\right\} .
$$

A Banach algebra is called left strongly Arens irregular if $\mathfrak{Z}_{t}^{(l)}\left(A^{* *}\right)=A$ (cf. [5]). The right topological centre of $A^{* *}$ is defined similarly. When $A$ is commutative, the left and right topological centres both coincide with the algebraic centre of $A^{* *}$ (with respect to either of the products).

Let $A$ be a Banach algebra and $X=\overline{\operatorname{Span}}^{\|\cdot\|} A^{*} \cdot A$. Then $A^{*}$ is a left Banach $X^{*}$-module with the action

$$
\langle\xi \odot f, a\rangle=\langle\xi, f \cdot a\rangle \quad\left(\xi \in X^{*}, f \in A^{*}, a \in A\right) .
$$

Moreover it is easily verified that $\xi \odot f=\widetilde{\xi} \cdot f$, where $\widetilde{\xi}$ is any HahnBanach extension of $\xi$ to a continuous linear functional on $A^{*}$.

For the definition of introverted subspaces of $A^{*}$ and the topological centres of their duals we refer to Işik-Pym-Ülger [29] or Lau-Dales [5]. The space $X=\overline{\operatorname{Span}}^{\|\cdot\|} A^{*} \cdot A$ is a left introverted subspace of $A^{*}$ and $X^{*}=\left(A^{* *}, \square\right) / X^{\circ}$ is a quotient algebra of $\left(A^{* *}, \square\right)$. We denote the quotient product on $X^{*}$ by $\square$. In this paper we shall also consider the special case where $A=A(G), A^{*}=V N(G)$, and $X=$ $U C_{2}(G)=\overline{\operatorname{Span}}^{\|\cdot\|} V N(G) \cdot A(G)=\overline{V N(G) \cdot A(G)}^{\|\cdot\|}$ (see [37, p. 4]). The $C^{*}$-algebra $U C_{2}(G)$ can be identified with the algebra of bounded 
uniformly continuous functions $U C(\widehat{G})$ if $G$ is abelian. If $G$ is amenable, then by the Cohen's factorization theorem, $U C_{2}(G)=V N(G) \cdot A(G)$. For more information on these spaces see Lau [33, 34].

For detailed properties of the Fourier algebra $A(G)$ and the FourierStieltjes algebra $B(G)$ we refer to [8]. For our purposes, we recall that if $T \in V N(G)$ and $u=\bar{g} * \check{f} \in A(G)$ where $f, g \in L^{2}(G)$, then $\langle T, u\rangle=$ $(T f \mid g)$. The function $T u \in A(G)$ is defined by $T u(x)=\left\langle T,{ }_{x^{-1}} \breve{u}\right\rangle$ for each $x \in G$.

\section{A Factorization Theorem for $V N(G)$}

Throughout this paper (unless otherwise stated) we shall assume that $G$ is a compact group whose local weight $b(G)$ has uncountable cofinality. For example, if $H$ is a non-trivial compact group and $\mathfrak{m}$ is a cardinal with uncountable cofinality, then the product group $H^{\mathfrak{m}}$ satisfies our requirements; another example is the semidirect product $G=\mathbf{T}^{\mathfrak{m}} \ltimes D_{n}$, where $\mathbf{T}$ is the circle group and $D_{n}$ is the dihedral group (whose rotations and reflections are automorphisms of the circle group). It is known that every compact, non-metrizable group contains a decreasing family $\left(N_{\alpha}\right)$ of closed normal subgroups such that $\cap_{\alpha} N_{\alpha}=\{e\}$ (see Lemma 3.1). The assumption that $b(G)$ has uncountable cofinality allows us to show the existence of 'sufficiently many' irreducible unitary representations of fixed dimension over the subgroups $N_{\alpha}$ (Lemma 3.3). We shall use the cluster points $C_{k l}^{\lambda}$ of the coordinate functions of these representations to obtain our factorization formula (23).

The following result (which we shall need in this section) is due to $\mathrm{Hu}$ [24, Proposition 4.3] which itself is a stronger version of an earlier result of Lau-Losert [37, Lemma 4.8]. Since there will be no fear of confusion, in what follows, we shall use $b(G)$ to denote also the initial ordinal $\mu$ such that $|\mu|=b(G)$.

Lemma 3.1. Let $G$ be a $\sigma$-compact, non-metrizable, locally compact group with unit element e. Let $\left\{O_{\alpha}: 0 \leq \alpha<b(G)\right\}$ be an open base at $e$. Then there exists a decreasing family $\left(N_{\alpha}\right)_{0 \leq \alpha \leq b(G)}$ of normal subgroups of $G$ (that is, $N_{\beta} \subset N_{\alpha}$ whenever $\alpha \leq \beta$ ) such that

(i) $N_{0}=G$ and $N_{b(G)}=\{e\}$,

(ii) $N_{\alpha+1} \subset N_{\alpha} \cap O_{\alpha}$ for all $0 \leq \alpha<b(G)$,

(iii) $N_{\alpha}$ is compact for each $\alpha>0$,

(iv) $N_{\alpha} / N_{\alpha+1}$ is metrizable but $N_{\alpha+1} \neq N_{\alpha}$ for all $\alpha<b(G)$;

(v) $N_{\gamma}=\cap_{\alpha<\gamma} N_{\alpha}$ for every limit ordinal $\gamma<b(G)$;

(vi) $b\left(N_{\alpha}\right)=b(G)$ for all $0 \leq \alpha<b(G)$.

For each $\alpha>0$, let $\lambda_{\alpha}$ be the normalized Haar measure on $N_{\alpha}$. As in $[37,24]$ we consider the family $\left(P_{\alpha}\right)_{0 \leq \alpha<b(G)}$ of orthogonal projections 
in $V N(G)$, where $P_{0}=0$ and $P_{\alpha}$ is defined by

$$
\left.P_{\alpha}: L^{2}(G) \longrightarrow L^{2}(G), \quad\left(P_{\alpha} f\right)(x)=\int_{N_{\alpha}} f\left(t^{-1} x\right) d \lambda_{\alpha}(t) \quad \text { (a.e. }\right) \text {. }
$$

If $f$ is continuous, then so is $P_{\alpha} f$, and we may assume (1) holds for all $x \in G$. It is easy to verify that $\left(P_{\alpha}\right)_{\alpha}$ is an increasing family of central orthogonal projections in $V N(G)$, that is, $P_{\alpha} P_{\beta}=P_{\beta} P_{\alpha}=P_{\alpha}$ for all $0 \leq \alpha \leq \beta<b(G)$.

Lemma 3.2. Let $G$ be a compact, non-metrizable group. Let $u \in A(G)$ and $u \cdot P_{\alpha} \in V N(G)$ be defined by the usual module action of $A(G)$ on its dual $V N(G)$. Then, for all $f \in L^{2}(G)$,

$$
\left.\left(u \cdot P_{\alpha}\right) f(x)=\int_{N_{\alpha}} u(t) f\left(t^{-1} x\right) d \lambda_{\alpha}(t) \quad \text { (a.e. }\right) .
$$

Proof. Let $f, g \in L^{2}(G)$, then

$$
\left\langle\left(u \cdot P_{\alpha}\right) f \mid g\right\rangle=\left\langle P_{\alpha},(\bar{g} * \check{f}) u\right\rangle=P_{\alpha}((\bar{g} * \check{f}) u)(e),
$$

thus by (1), we can write

$$
\begin{aligned}
\left(\left(u \cdot P_{\alpha}\right) f \mid g\right) & =\int_{N_{\alpha}} u(t)(\bar{g} * \check{f})(t) d \lambda_{\alpha}(t) \\
& =\int_{N_{\alpha}} \int_{G} u(t) \overline{g(y)} \check{f}\left(y^{-1} t\right) d y d \lambda_{\alpha}(t) \\
& =\int_{G} \overline{g(y)} \int_{N_{\alpha}} u(t) f\left(t^{-1} y\right) d \lambda_{\alpha}(t) d y=(F \mid g),
\end{aligned}
$$

where $F \in L^{2}(G)$ is defined (a.e.) by

$$
F(x)=\int_{N_{\alpha}} u(t) f\left(t^{-1} x\right) d \lambda_{\alpha}(t) .
$$

Therefore $\left(u \cdot P_{\alpha}\right) f=F$.

For each $\alpha<b(G)$, let $\widehat{N_{\alpha}}$ be the set of all equivalence classes of irreducible unitary representations of $N_{\alpha}$. Since for each $\alpha<b(G), N_{\alpha}$ is compact, representations in $\widehat{N_{\alpha}}$ are all finite-dimensional. We note that

$$
\left|\widehat{N_{\alpha}}\right|=b\left(N_{\alpha}\right)=b(G)
$$

by Hewitt-Ross [21, Theorem 28.2 and Remark 28.58(b)]. Let $\widehat{G}=$ $\left\{V_{\gamma}: \gamma<b(G)\right\}$, with $V_{0}$ the trivial one-dimensional representation defined by $V_{0}(t)=1(t \in G)$. For every $0<\alpha<b(G)$, we define

$$
\widehat{G}_{\alpha}=\left\{V_{\gamma} \in \widehat{G}: 0 \leq \gamma<\alpha\right\} .
$$

Let $\left\{\widetilde{U}_{\alpha}\right\}_{\alpha<b(G)}$ be a family of irreducible unitary representations of $G$. For each $\beta<b(G)$ and $\gamma<b(G)$, the finite-dimensional representation $\left.\left(V_{\gamma} \otimes \widetilde{U}_{\beta}\right)\right|_{N_{\beta}}$ can be decomposed (in a unique way up to 
equivalence) into a finite direct sum of irreducible unitary representations of $N_{\beta}$ (cf. Folland [15, Theorem 5.2]). We denote by $\mathcal{P}_{\gamma, \beta}$ the set consisting of representations appearing in such a decomposition. Similarly, when restricted to $N_{\alpha}(\alpha>\beta)$, every representation in $\mathcal{P}_{\gamma, \beta}$ can be written as a finite direct sum of irreducible unitary representations of $N_{\alpha}$. Let $\mathcal{R}_{\gamma, \beta}^{\alpha}$ consists of all irreducible unitary representations of $N_{\alpha}$ appearing in such a decomposition of members of $\mathcal{P}_{\gamma, \beta}$.

Lemma 3.3. Let $G$ be a compact group such that $b(G)$ has uncountable cofinality. Then there exist two families of irreducible unitary representations $\left\{U_{\alpha}\right\}_{\alpha<b(G)},\left\{\widetilde{U}_{\alpha}\right\}_{\alpha<b(G)}$, and there exist $n, n^{\prime} \in \mathbf{N}$ such that for each $\alpha<b(G)$ we have

(i) $U_{\alpha} \in \widehat{N_{\alpha}}$ and $\widetilde{U}_{\alpha} \in \widehat{G}$;

(ii) $\widetilde{U}_{\alpha}$ is an extension of $U_{\alpha}$;

(iii) $U_{\alpha} \notin \bigcup_{\beta, \gamma<\alpha} \mathcal{R}_{\gamma, \beta}^{\alpha}$;

(iv) $\operatorname{dim} U_{\alpha}=n$ and $\operatorname{dim} \widetilde{U}_{\alpha}=n^{\prime}$ for $\alpha>0$.

Proof. Let $U_{0}=\widetilde{U}_{0}=V_{0}$ be the trivial one-dimensional representation of $G$. Let $\alpha<b(G)$ and suppose that $U_{\beta}$ and $\widetilde{U}_{\beta}$ are chosen for every $\beta<\alpha$. Then

$$
\left|\bigcup_{\beta, \gamma<\alpha} \mathcal{R}_{\gamma, \beta}^{\alpha}\right| \leq \aleph_{0} \cdot|\alpha|^{2}<b(G)=\left|\widehat{N}_{\alpha}\right| .
$$

Using transfinite induction, we can choose a representation $U_{\alpha} \in \widehat{N_{\alpha}}$ such that

$$
U_{\alpha} \notin \bigcup_{\beta, \gamma<\alpha} \mathcal{R}_{\gamma, \beta}^{\alpha} .
$$

By Hewitt-Ross [21, Theorem 27.46] we can extend $U_{\alpha}$ to an irreducible unitary representation of $G$, which we denote by $\widetilde{U}_{\alpha}$.

Since each $U_{\alpha}$ has finite dimension, if we define $E_{m}=\left\{\alpha: \operatorname{dim} U_{\alpha}=\right.$ $m\}$, then $\cup_{m=1}^{\infty} E_{m}=\{\alpha: \alpha<b(G)\}$. It follows from our assumptions that for at least one $m$, say $m=n, E_{n}$ has cardinality $b(G)$ (cf. Jech [30, Lemma 3.6]). Thus by replacing the family of subgroups $\left\{N_{\alpha}\right\}(0<$ $\alpha<b(G))$ and its associated family of representation $\left\{U_{\alpha}\right\}$ with those that are indexed by the set $E_{n}$, we obtain a family of irreducible unitary representations, which for simplicity we still denote by $\left(U_{\alpha}\right)_{\alpha<b(G)}$, such that $\operatorname{dim} U_{\alpha}=n$ for all $\alpha<b(G)$. Having fixed the dimensions of the representations $U_{\alpha}$, we can repeat this argument for the family $\left\{\widetilde{U}_{\alpha}\right\}$, and therefore, we may assume as well that $\operatorname{dim} \widetilde{U}_{\alpha}=n^{\prime}$, where $n^{\prime} \geq n$ is a fixed positive integer.

Representations $U_{\alpha}$ and $\widetilde{U}_{\alpha}$ of dimensions independent of $\alpha$ play an important role in our factorization Theorem 3.11. In the case that cofinality of $b(G)$ is countable, the situation seems more complicated, since in this case we have been unable to fix the dimensions of the 
representations. In fact, the only case so far known is the special case of a countable product $G=G_{0} \times \prod_{i=1}^{\infty} G_{i}$ where each $G_{i}$ is a compact nontrivial group for $i \geq 1$, dealt with in Lau-Losert [38], in which case there exits a countable filtration $\left\{N_{k}\right\}$, where $N_{k}=\prod_{i=k}^{\infty} G_{i}$.

We shall write $H_{\alpha}$ and $\widetilde{H}_{\alpha}$ for the representation spaces of $U_{\alpha}$ and $\widetilde{U}_{\alpha}$, respectively. If $\left\{e_{1}, \ldots, e_{n^{\prime}}\right\}$ is an orthonormal basis for $\widetilde{H}_{\alpha}$, we shall always assume that $\left\{e_{1}, \ldots, e_{n}\right\}$ form a basis for the subspace $H_{\alpha}$. In such an orthonormal basis, we can write $\widetilde{U}_{\alpha}=\left(\tilde{u}_{i j}^{\alpha}\right)_{1 \leq i, j \leq n^{\prime}}$, where $\tilde{u}_{i j}^{\alpha}$ are the coordinate functions of $\widetilde{U}_{\alpha}$. Similarly, we write $U_{\alpha}=\left(u_{i j}^{\alpha}\right)_{1 \leq i, j \leq n}$ and more generally $V=\left(v_{p^{\prime} q^{\prime}}\right)_{1 \leq p^{\prime}, q^{\prime} \leq \operatorname{dim} V_{\gamma}}$ if $V \in \widehat{G}$.

To avoid unnecessary constants in the statements of our results, we substitute each representation $\widetilde{U}_{\alpha}$ with the representation $n \widetilde{U}_{\alpha}$ (where $n$ is the fixed dimension of the representations $U_{\alpha}$ ). For simplicity, we shall continue to denote these 'normalized' representations and their coordinate functions by $\widetilde{U}_{\alpha}$ and $\tilde{u}_{i j}^{\alpha}$. We remark that since $\widetilde{U}_{\alpha}$ is a unitary extension of $U_{\alpha}$, if $t \in N_{\alpha}$, then $\widetilde{U}_{\alpha}(t)=\left(\tilde{u}_{k l}^{\alpha}(t)\right)$ is a block matrix, whose upper left corner is the $n \times n$ matrix $\left(n u_{i j}^{\alpha}(t)\right)$ and in its lower left and upper right corners have zero entries:

$$
\widetilde{U}_{\alpha}(t)=\left(\begin{array}{cccccc}
n u_{11}^{\alpha}(t) & \cdots & n u_{1 n}^{\alpha}(t) & 0 & \cdots & 0 \\
\vdots & & \vdots & \vdots & & \vdots \\
n u_{n 1}^{\alpha}(t) & \cdots & n u_{n n}^{\alpha}(t) & 0 & \cdots & 0 \\
0 & \cdots & 0 & \tilde{u}_{n(n+1)}^{\alpha}(t) & \cdots & \tilde{u}_{n n^{\prime}}^{\alpha}(t) \\
\vdots & & \vdots & \vdots & & \vdots \\
0 & \cdots & 0 & \tilde{u}_{n^{\prime}(n+1)}^{\alpha}(t) & \cdots & \tilde{u}_{n^{\prime} n^{\prime}}^{\alpha}(t)
\end{array}\right)_{n^{\prime} \times n^{\prime}} .
$$

We note that since $G$ is compact, we have $\tilde{u}_{k l}^{\alpha} \in A(G)$ for all $\alpha<b(G)$ and all $k, l \in\left\{1,2, \ldots, n^{\prime}\right\}$. In addition, by Eymard [8, Lemma 2.14], the family of coordinate functions $\tilde{u}_{k l}^{\alpha}$ is bounded, in fact

$$
\left\|\tilde{u}_{k l}^{\alpha}\right\|_{A(G)}=n\left\|\left(\widetilde{U}_{\alpha}(\cdot) e_{l} \mid e_{k}\right)\right\|_{A(G)} \leq n\left\|e_{l}\right\|\left\|e_{k}\right\|=n .
$$

Lemma 3.4. Let $G$ be a compact group such that $b(G)$ has uncountable cofinality. Let $V \in \widehat{G}$ be arbitrary and let $\bar{V}$ be the conjugate representation of $V$. Let $\alpha$ be large enough so that $V, \bar{V} \in \widehat{G}_{\alpha}$. If $\beta \neq \alpha$, $1 \leq i, k \leq n^{\prime}, 1 \leq j, l \leq n$ and $1 \leq p^{\prime}, q^{\prime} \leq \operatorname{dim} V$, then

$$
\int_{N_{\beta}} \tilde{u}_{i j}^{\alpha}(t) \overline{\tilde{u}}_{k l}^{\beta}(t) \bar{v}_{p^{\prime} q^{\prime}}(t) d \lambda_{\beta}(t)=0 .
$$

Proof. First we assume that $\alpha>\beta$ and therefore $N_{\alpha} \subset N_{\beta}$. Note that $\tilde{u}_{k l}^{\beta} v_{p^{\prime} q^{\prime}}$ is a coordinate function of $V \otimes \widetilde{U}_{\beta}$. Let

$$
\begin{aligned}
\left.\widetilde{U}_{\alpha}\right|_{N_{\beta}} & =W_{1} \oplus \cdots \oplus W_{s}, \\
\left.\left(V \otimes \widetilde{U}_{\beta}\right)\right|_{N_{\beta}} & =R_{1} \oplus \cdots \oplus R_{s^{\prime}},
\end{aligned}
$$


be the decompositions of $\left.\widetilde{U}_{\alpha}\right|_{N_{\beta}}$ and $\left.\left(V \otimes \widetilde{U}_{\beta}\right)\right|_{N_{\beta}}$ into direct sums of irreducible unitary representations of $N_{\beta}$. Let $\left\{e_{i j}: 1 \leq i \leq s, 1 \leq j \leq\right.$ $\left.n_{i}\right\}$ be an orthonormal basis for $\widetilde{H}_{\alpha}$ corresponding to the decomposition (6). Let $E_{i}=\operatorname{Span}\left\{e_{i j}: 1 \leq j \leq n_{i}\right\}$. Since $H_{\alpha} \subset \widetilde{H}_{\alpha}$ and $H_{\alpha} \cap E_{i}$ is invariant under $U_{\alpha}$, it follows from the irreducibility of $U_{\alpha}$ that either $H_{\alpha} \cap E_{i}=\{0\}$ or $H_{\alpha} \subset E_{i}$. Hence, without loss of generality, we may assume that $H_{\alpha} \subset E_{1}$. By a similar argument, we may assume that $U_{\alpha}$ is contained in $\left.W_{1}\right|_{N_{\alpha}}$, in fact, if $\left.W_{1}\right|_{N_{\alpha}}=W_{11} \oplus \cdots \oplus W_{1 q}$ is the decomposition of $\left.W_{1}\right|_{N_{\alpha}}$ into irreducible components, with $F_{1}, \ldots, F_{q}$ the corresponding subspaces of $E_{1}$, then each $H_{\alpha} \cap F_{j}$ is an invariant subspace of $H_{\alpha}$ under $W_{1}(t)=U_{\alpha}(t)$ for $t \in N_{\alpha}$. Hence the irreducibility of $U_{\alpha}$ implies that either $H_{\alpha} \cap F_{j}=\{0\}$ or else $H_{\alpha}=F_{j}$. Since $H_{\alpha}$ cannot have trivial intersection with all the $F_{j}$, we must $H_{\alpha}=F_{j}$ and hence $U_{\alpha}=W_{1 j}$ for some $j$.

For $1 \leq i \leq n^{\prime}, 1 \leq j \leq n$, and $t \in N_{\beta}$ we have

$$
\begin{aligned}
\tilde{u}_{i j}^{\alpha}(t)=\left(\widetilde{U}_{\alpha}(t) e_{j} \mid e_{i}\right) & =\left(\widetilde{U}_{\alpha}(t)\left(\sum_{p=1}^{n_{1}} \xi_{p}^{j} e_{1 p}\right) \mid \sum_{r=1}^{s} \sum_{q=1}^{n_{r}} \xi_{r q}^{i} e_{r q}\right) \\
& =\sum_{p=1}^{n_{1}} \sum_{r=1}^{s} \sum_{q=1}^{n_{r}} \xi_{p}^{j} \overline{\xi_{r q}^{i}}\left(\widetilde{U}_{\alpha}(t) e_{1 p} \mid e_{r q}\right) \\
(\operatorname{by}(6)) & =\sum_{p=1}^{n_{1}} \sum_{q=1}^{n_{1}} \xi_{p}^{j} \overline{\xi_{1 q}^{i}}\left(\widetilde{U}_{\alpha}(t) e_{1 p} \mid e_{1 q}\right) \\
& =\sum_{p=1}^{n_{1}} \sum_{q=1}^{n_{1}} \xi_{p}^{j} \overline{\xi_{1 q}^{i}}\left(W_{1}(t) e_{1 p} \mid e_{1 q}\right),
\end{aligned}
$$

since by $(6),\left(\widetilde{U}_{\alpha}(t) e_{1 p} \mid e_{r q}\right)=0$ for $r \geq 2$, and $\left.\widetilde{U}_{\alpha}(t)\right|_{E_{1}}=W_{1}(t)$ when $t \in N_{\beta}$.

On $N_{\beta}$ the functions $\tilde{u}_{k l}^{\beta} v_{p^{\prime} q^{\prime}}$ are linear combinations of coordinate functions of the representations $R_{r}\left(r=1, \ldots, s^{\prime}\right)$. However $R_{r} ¥ W_{1}$ for every $r$, since by our assumptions $U_{\alpha} \notin \bigcup_{\beta, \gamma<\alpha} \mathcal{R}_{\gamma, \beta}^{\alpha}$. Thus each $\tilde{u}_{k l}^{\beta} v_{p^{\prime} q^{\prime}}$ is a linear combination of coordinate functions of irreducible unitary representations of $N_{\beta}$ none of which are equivalent to $W_{1}$. Thus by (8) and by the orthogonality relations in Hewitt-Ross [21, Theorem 27.19], the equation (5) holds.

It remains to assume that $\beta>\alpha$. In this case $N_{\beta} \subset N_{\alpha}$. We choose $\gamma$ so that $\bar{V}=V_{\gamma}$. Then by the choice of $U_{\beta}$, we have $U_{\beta} \notin \mathcal{R}_{\gamma, \alpha}^{\beta}$. The functions $\tilde{u}_{i j}^{\alpha} \bar{v}_{p^{\prime} q^{\prime}}$ are coordinate functions of the representation $\bar{V} \otimes \widetilde{U}_{\alpha}$, which on $N_{\beta}$ breaks into a direct sum of irreducible unitary representations, all in $\mathcal{R}_{\gamma, \alpha}^{\beta}$. Thus on $N_{\beta}$, the functions $\tilde{u}_{i j}^{\alpha} \bar{v}_{p^{\prime} q^{\prime}}$ are linear combinations of the coordinate functions of representations in $\mathcal{R}_{\gamma, \alpha}^{\beta}$. These latter functions are orthogonal to $\tilde{u}_{k l}^{\beta}$ on $N_{\beta}$ (since for 
$1 \leq l \leq n$ we have $\tilde{u}_{k l}^{\beta}=0$ on $N_{\beta}$ if $k>n$, and moreover $\tilde{u}_{k l}^{\beta}=n u_{k l}^{\beta}$ if $1 \leq k \leq n)$, and therefore so are the functions $\tilde{u}_{i j}^{\alpha} \bar{v}_{p^{\prime} q^{\prime}}$. Hence

$$
0=\int_{N_{\beta}} \tilde{u}_{k l}^{\beta}(t) \overline{\tilde{u}_{i j}^{\alpha}(t) \bar{v}_{p^{\prime} q^{\prime}}(t)} d \lambda_{\beta}(t)=\overline{\int_{N_{\beta}} \tilde{u}_{i j}^{\alpha}(t) \overline{\tilde{u}}_{k l}^{\beta}(t) \bar{v}_{p^{\prime} q^{\prime}}(t) d \lambda_{\beta}(t),}
$$

which completes the proof of the lemma.

Lemma 3.5. If $1 \leq i, j, k \leq n$ and $1 \leq l \leq n^{\prime}$, then

$$
\left(\tilde{u}_{i j}^{\alpha} \cdot P_{\alpha}\right)\left(\tilde{u}_{k l}^{\beta} \cdot P_{\beta}\right)=\delta_{\alpha \beta} \delta_{j k}\left(\tilde{u}_{i l}^{\alpha} \cdot P_{\alpha}\right) .
$$

Proof. It suffices to show that the actions of both sides of (9) on an arbitrary element $f \in L^{2}(G)$ are the same.

If $l>n$, then by Lemma 3.2, and the fact that for $1 \leq i, k \leq n$, $\tilde{u}_{i l}^{\alpha}(t)=0\left(t \in N_{\alpha}\right)$ and $\tilde{u}_{k l}^{\beta}(s)=0\left(s \in N_{\beta}\right)$ (see (4)), it follows that the action of both sides of $(9)$ on $f$ will be equal to 0 and our claim follows immediately.

If remains to consider the case that $1 \leq l \leq n$. Let $\alpha \leq \beta$, then $N_{\beta} \subset N_{\alpha}$. By Lemma 3.2, we have

$$
\begin{aligned}
\left(( \tilde { u } _ { i j } ^ { \alpha } \cdot P _ { \alpha } ) \left(\tilde{u}_{k l}^{\beta}\right.\right. & \left.\left.\cdot P_{\beta}\right) f\right)(x)=\left(\tilde{u}_{i j}^{\alpha} \cdot P_{\alpha}\right)\left(\left(\tilde{u}_{k l}^{\beta} \cdot P_{\beta}\right) f\right)(x) \\
& =\int_{N_{\alpha}} \tilde{u}_{i j}^{\alpha}(t)\left[\left(\tilde{u}_{k l}^{\beta} \cdot P_{\beta}\right) f\right]\left(t^{-1} x\right) d \lambda_{\alpha}(t) \\
& =\int_{N_{\alpha}} \tilde{u}_{i j}^{\alpha}(t) \int_{N_{\beta}} \tilde{u}_{k l}^{\beta}(s) f\left(s^{-1} t^{-1} x\right) d \lambda_{\beta}(s) d \lambda_{\alpha}(t) \\
& =\int_{N_{\alpha}} n u_{i j}^{\alpha}(t) \int_{N_{\beta}} n u_{k l}^{\beta}(s) f\left(s^{-1} t^{-1} x\right) d \lambda_{\beta}(s) d \lambda_{\alpha}(t) \\
& =n^{2} \int_{N_{\beta}} \int_{N_{\alpha}} u_{i j}^{\alpha}(t) u_{k l}^{\beta}(s) f\left(s^{-1} t^{-1} x\right) d \lambda_{\alpha}(t) d \lambda_{\beta}(s) \\
& =n^{2} \int_{N_{\beta}} \int_{N_{\alpha}} u_{i j}^{\alpha}\left(y s^{-1}\right) u_{k l}^{\beta}(s) f\left(y^{-1} x\right) d \lambda_{\alpha}(y) d \lambda_{\beta}(s),
\end{aligned}
$$

where the fourth equality was obtained since for $1 \leq i, j, k, l \leq n$ we have $\tilde{u}_{i j}^{\alpha}(t)=n u_{i j}^{\alpha}(t)$ if $t \in N_{\alpha}$, and $\tilde{u}_{k l}^{\beta}(s)=n u_{k l}^{\beta}(s)$ if $s \in N_{\beta}$; the last equality was obtained by the change of variable $t=y s^{-1}$ in $N_{\alpha}$. However,

$$
u_{i j}^{\alpha}\left(y s^{-1}\right)=\left(U_{\alpha}\left(y s^{-1}\right) e_{j} \mid e_{i}\right)=\left(U_{\alpha}(s)^{*} e_{j} \mid U_{\alpha}(y)^{*} e_{i}\right)=\sum_{r=1}^{n} \bar{u}_{j r}^{\alpha}(s) u_{i r}^{\alpha}(y) .
$$

Therefore,

$$
\begin{aligned}
& \left(\left(\tilde{u}_{i j}^{\alpha} \cdot P_{\alpha}\right)\left(\tilde{u}_{k l}^{\beta} \cdot P_{\beta}\right) f\right)(x) \\
& \quad=n^{2} \sum_{r=1}^{n}\left(\int_{N_{\beta}} \bar{u}_{j r}^{\alpha}(s) u_{k l}^{\beta}(s) d \lambda_{\beta}(s)\right)\left(\int_{N_{\alpha}} u_{i r}^{\alpha}(y) f\left(y^{-1} x\right) d \lambda_{\alpha}(y)\right) .
\end{aligned}
$$


By Lemma 3.4 (in which we take $V$ to be the trivial one-dimensional representation $V_{0}$ ) and by the orthogonality relations in Hewitt-Ross [21, Theorems 27.19] we have

$$
\int_{N_{\beta}} \bar{u}_{j r}^{\alpha}(s) u_{k l}^{\beta}(s) d \lambda_{\beta}(s)=\frac{1}{n^{2}} \int_{N_{\beta}} \overline{\tilde{u}}_{j r}^{\alpha}(s) \tilde{u}_{k l}^{\beta}(s) d \lambda_{\beta}(s)=\frac{1}{n} \delta_{\alpha \beta} \delta_{j k} \delta_{r l} .
$$

Thus,

$$
\begin{aligned}
\left(\left(\tilde{u}_{i j}^{\alpha} \cdot P_{\alpha}\right)\left(\tilde{u}_{k l}^{\beta} \cdot P_{\beta}\right) f\right)(x) & =n \delta_{\alpha \beta} \delta_{j k} \int_{N_{\alpha}} u_{i l}^{\alpha}(y) f\left(y^{-1} x\right) d \lambda_{\alpha}(y) \\
& =\delta_{\alpha \beta} \delta_{j k} \int_{N_{\alpha}} \tilde{u}_{i l}^{\alpha}(y) f\left(y^{-1} x\right) d \lambda_{\alpha}(y) \\
& =\delta_{\alpha \beta} \delta_{j k}\left(\tilde{u}_{i l}^{\alpha} \cdot P_{\alpha}\right) f(x) .
\end{aligned}
$$

It follows that $\left(\tilde{u}_{i j}^{\alpha} \cdot P_{\alpha}\right)\left(\tilde{u}_{k l}^{\beta} \cdot P_{\beta}\right)=\delta_{\alpha \beta} \delta_{j k}\left(\tilde{u}_{i l}^{\alpha} \cdot P_{\alpha}\right)$.

The argument for the case that $\beta<\alpha$ is similar.

Lemma 3.6. For every $\alpha<b(G)$ and $1 \leq k, l \leq n,\left(\tilde{u}_{k l}^{\alpha} \cdot P_{\alpha}\right)^{*}=\tilde{u}_{l k}^{\alpha} \cdot P_{\alpha}$. In particular, $\tilde{u}_{k k}^{\alpha} \cdot P_{\alpha}$ is an orthogonal projection.

Proof. If $f, g \in L^{2}(G)$ are arbitrary, then using Lemma 3.2 we have

$$
\begin{aligned}
\left(\left(\tilde{u}_{k l}^{\alpha} \cdot P_{\alpha}\right) f \mid g\right) & =\int_{G} \bar{g}(x)\left(\left(\tilde{u}_{k l}^{\alpha} \cdot P_{\alpha}\right) f\right)(x) d x \\
& =\int_{G} \bar{g}(x) \int_{N_{\alpha}} \tilde{u}_{k l}^{\alpha}(t) f\left(t^{-1} x\right) d \lambda_{\alpha}(t) d x .
\end{aligned}
$$

Changing the order of the integrations and making the substitution $x \rightarrow t x$, we have

$$
\begin{aligned}
\left(\left(\tilde{u}_{k l}^{\alpha} \cdot P_{\alpha}\right) f \mid g\right) & =\int_{N_{\alpha}} \tilde{u}_{k l}^{\alpha}(t) \int_{G} \bar{g}(t x) f(x) d x d \lambda_{\alpha}(t) \\
& =\int_{G} f(x) \int_{N_{\alpha}} \tilde{u}_{k l}^{\alpha}\left(t^{-1}\right) \bar{g}\left(t^{-1} x\right) d \lambda_{\alpha}(t) d x \\
& =\int_{G} f(x) \int_{N_{\alpha}} \overline{\tilde{u}_{l k}^{\alpha}}(t) \bar{g}\left(t^{-1} x\right) d \lambda_{\alpha}(t) d x \\
& =\int_{G} f(x) \overline{\left(\left(\tilde{u}_{l k}^{\alpha} \cdot P_{\alpha}\right) g\right)}(x) d x \\
& =\left(f \mid\left(\tilde{u}_{l k}^{\alpha} \cdot P_{\alpha}\right) g\right) .
\end{aligned}
$$

Therefore, $\left(\tilde{u}_{k l}^{\alpha} \cdot P_{\alpha}\right)^{*}=\tilde{u}_{l k}^{\alpha} \cdot P_{\alpha}$. Moreover, by Lemma 3.5, we have $\left(\tilde{u}_{k k}^{\alpha} \cdot P_{\alpha}\right)^{2}=\tilde{u}_{k k}^{\alpha} \cdot P_{\alpha}$.

In preparation for our next lemma, we note that if $X$ is a Banach space and if $\sum_{\varphi \in I} \varphi_{\alpha}$ is a $w^{*}$-unconditionally convergent series in $X^{*}$ (that is, $\sum_{\alpha \in I}\left|\left\langle\varphi_{\alpha}, x\right\rangle\right|<\infty$ for all $x \in X$ ) whose partial sums are uniformly norm-bounded, then these partial sums form a $w^{*}$-Cauchy net in $X^{*}$, and so the series is $w^{*}$-convergent in $X^{*}$ by Alaoglu's theorem. 
Lemma 3.7. Let $\mathcal{M} \subset \mathcal{B}(H)$ be a von Neumann algebra. Consider a bounded family $\left(T_{\alpha}\right)_{\alpha \in I}$ of elements in $\mathcal{M}$ such that for all $\alpha \in I$, $\left\|T_{\alpha}\right\| \leq r$ (for some $r>0$ ), and let $\left(P_{\alpha}\right)_{\alpha \in I}$ and $\left(Q_{\alpha}\right)_{\alpha \in I}$ be two families of orthogonal projections in $\mathcal{M}$ such that

$$
P_{\alpha} P_{\beta}=Q_{\alpha} Q_{\beta}=0, \quad \text { if } \alpha \neq \beta \text {. }
$$

Then the $w^{*}$-limit

$$
\sum_{\alpha \in I} P_{\alpha} T_{\alpha} Q_{\alpha}
$$

exists in $\mathcal{M}$, and defines a $w^{*}$-unconditionally convergent series. Moreover, the norm of the sum does not exceed $r$.

Proof. Fix $\xi, \eta \in H$. Then we have

$$
\begin{aligned}
\sum_{\alpha \in I}\left|\left(P_{\alpha} T_{\alpha} Q_{\alpha} \xi \mid \eta\right)\right| & =\sum_{\alpha \in I}\left|\left(T_{\alpha} Q_{\alpha} \xi \mid P_{\alpha} \eta\right)\right| \\
& \leq r \sum_{\alpha \in I}\left\|Q_{\alpha} \xi\right\|\left\|P_{\alpha} \eta\right\| \\
& \leq r\left(\sum_{\alpha \in I}\left\|Q_{\alpha} \xi\right\|^{2}\right)^{\frac{1}{2}}\left(\sum_{\alpha \in I}\left\|P_{\alpha} \eta\right\|^{2}\right)^{\frac{1}{2}} \\
& \leq r\|\xi\|\|\eta\|,
\end{aligned}
$$

where the last line follows of course from Bessel's inequality. In particular, this entails that the partial sums $\sum_{\alpha \in F} P_{\alpha} T_{\alpha} Q_{\alpha}$, where $F \subset I$ is finite, are uniformly norm-bounded.

Now consider an arbitrary element $\rho \in \mathcal{B}(H)_{*}=\mathcal{T}(H)=H \otimes_{\gamma} H$ (the latter denotes the projective Banach space tensor product). We have a series expansion $\rho=\sum_{i=1}^{\infty} \xi_{i} \otimes \eta_{i}$, where for all $i \in \mathbf{N}, \xi_{i}, \eta_{i} \in H$, and $\sum_{i=1}^{\infty}\left\|\xi_{i}\right\|\left\|\eta_{i}\right\|<\infty$. We note, thanks to this last property and the estimation (10), for every finite subset $F$ in $I$, the limit

$$
\sum_{i=1}^{\infty} \sum_{\alpha \in F}\left|\left\langle P_{\alpha} T_{\alpha} Q_{\alpha}, \xi_{i} \otimes \eta_{i}\right\rangle\right|
$$

exists uniformly on $\mathfrak{P}(I)_{\text {fin }}$ (that is, the collection of all finite subsets of $I$ ). Hence by the classical theorem on the interchange of limits, we 
finally get

$$
\begin{aligned}
\sum_{\alpha \in I}\left|\left\langle P_{\alpha} T_{\alpha} Q_{\alpha}, \rho\right\rangle\right| & =\sum_{\alpha \in I}\left|\left\langle P_{\alpha} T_{\alpha} Q_{\alpha}, \sum_{i=1}^{\infty} \xi_{i} \otimes \eta_{i}\right\rangle\right| \\
& \leq \sum_{\alpha \in I} \sum_{i=1}^{\infty}\left|\left\langle P_{\alpha} T_{\alpha} Q_{\alpha}, \xi_{i} \otimes \eta_{i}\right\rangle\right| \\
& =\sum_{i=1}^{\infty} \sum_{\alpha \in I}\left|\left\langle P_{\alpha} T_{\alpha} Q_{\alpha}, \xi_{i} \otimes \eta_{i}\right\rangle\right| \\
& \stackrel{(10)}{\leq} r \sum_{i=1}^{\infty}\left\|\xi_{i}\right\|\left\|\eta_{i}\right\| \\
& <\infty .
\end{aligned}
$$

This shows that the sum $\sum_{\alpha \in I} P_{\alpha} T_{\alpha} P_{\alpha}$ is $w^{*}$-unconditionally convergent in $\mathcal{B}(H)$. We deduce the asserted $w^{*}$-convergence of the sum in $\mathcal{B}(H)$, and hence in $\mathcal{M}$. Finally, due to inequality (10), the norm of the sum is at most $r$.

Lemma 3.8. For each $Q \in V N(G), 1 \leq k, l \leq n$, and $1 \leq r \leq n^{\prime}$, the sum

$$
P_{k l r}=\frac{1}{n^{6}} \sum_{\beta<b(G)}\left(\tilde{u}_{k r}^{\beta} \cdot P_{\beta}\right)\left(\tilde{u}_{r 1}^{\beta} \cdot Q\right)\left(\tilde{u}_{1 l}^{\beta} \cdot P_{\beta}\right)
$$

is $w^{*}$-convergent in $V N(G)$.

Proof. By Lemma 3.5,

$$
\begin{aligned}
& \tilde{u}_{k r}^{\beta} \cdot P_{\beta}=\left(\tilde{u}_{k k}^{\beta} \cdot P_{\beta}\right)\left(\tilde{u}_{k r}^{\beta} \cdot P_{\beta}\right), \\
& \tilde{u}_{1 l}^{\beta} \cdot P_{\beta}=\left(\tilde{u}_{1 l}^{\beta} \cdot P_{\beta}\right)\left(\tilde{u}_{l l}^{\beta} \cdot P_{\beta}\right) .
\end{aligned}
$$

If we define $T_{\beta}=\left(\tilde{u}_{k r}^{\beta} \cdot P_{\beta}\right)\left(\tilde{u}_{r 1}^{\beta} \cdot Q\right)\left(\tilde{u}_{1 l}^{\beta} \cdot P_{\beta}\right)$, then

$$
P_{k l r}=\frac{1}{n^{6}} \sum_{\beta<b(G)}\left(\tilde{u}_{k k}^{\beta} \cdot P_{\beta}\right) T_{\beta}\left(\tilde{u}_{l l}^{\beta} \cdot P_{\beta}\right) .
$$

By Lemma 3.6, $\tilde{u}_{k k}^{\beta} \cdot P_{\beta}$ and $\tilde{u}_{l l}^{\beta} \cdot P_{\beta}$ are orthogonal projections in $V N(G)$. Clearly, $T_{\beta} \in V N(G)$ and $\left\|T_{\beta}\right\| \leq n^{3}\|Q\|$ for all $\beta<b(G)$, since $\left\|P_{\beta}\right\| \leq 1$ and $\left\|\tilde{u}_{p q}^{\beta}\right\| \leq n$ for $1 \leq p, q \leq n^{\prime}$ (see Eymard [8, Lemma 2.14] and our discussion preceding Lemma 3.4). The convergence of the sum in $w^{*}$-topology now follows from Lemma 3.7.

Using (11), for each $1 \leq k, l \leq n$, we define

$$
P_{k l}=\sum_{r=1}^{n^{\prime}} P_{k l r} .
$$

Now we are ready to prove our first factorization theorem. 
Theorem 3.9. Let $G$ be a compact group such that $b(G)$ has uncountable cofinality. For each $1 \leq k, l \leq n$, let $C_{k l}$ be a cluster point of the net $\left(\overline{\tilde{u}}_{k l}^{\alpha}\right)_{\alpha<b(G)}$ in $A(G)^{* *}$. Then, for every $Q \in V N(G)$, we have

$$
Q=\sum_{k=1}^{n} \sum_{l=1}^{n} C_{k l} \cdot P_{k l} .
$$

Proof. To prove the theorem we show that the actions of both sides of (13) on an arbitrary element $v$ of $A(G)$ are equal. Since $G$ is compact, an arbitrary element of $A(G)$ is a finite linear combination of coordinate functions of irreducible unitary representations of $G$. So it suffices to assume that

$$
v(s)=v_{i j}(s)=\left(V_{\gamma}(s) e_{j} \mid e_{i}\right) \quad(s \in G),
$$

where $V_{\gamma} \in \widehat{G}$, $\operatorname{dim} V_{\gamma}=d$, and $\left\{e_{1}, \ldots, e_{d}\right\}$ is the standard orthonormal basis of $\mathbf{C}^{d}$.

Without loss of generality we can assume that $\overline{\tilde{u}}_{k l}^{\alpha} \rightarrow C_{k l}$ in the $w^{*}$-topology; hence by Eymard [8, Proposition 3.17] we can write

$$
\begin{aligned}
& \left\langle C_{k l} \cdot P_{k l r}, v_{i j}\right\rangle=\left\langle C_{k l}, P_{k l r} \cdot v_{i j}\right\rangle \\
& =\frac{1}{n^{6}} \lim _{\alpha}\left\langle\sum_{\beta<b(G)}\left(\tilde{u}_{k r}^{\beta} \cdot P_{\beta}\right)\left(\tilde{u}_{r 1}^{\beta} \cdot Q\right)\left(\tilde{u}_{1 l}^{\beta} \cdot P_{\beta}\right), v_{i j} \overline{\tilde{u}}_{k l}^{\alpha}\right\rangle \\
& =\frac{1}{n^{6}} \lim _{\alpha} \sum_{\beta<b(G)}\left[\left\{\left(\tilde{u}_{k r}^{\beta} \cdot P_{\beta}\right)\left(\tilde{u}_{r 1}^{\beta} \cdot Q\right)\left(\tilde{u}_{1 l}^{\beta} \cdot P_{\beta}\right)\right\}\left(v_{i j} \overline{\tilde{u}}_{k l}^{\alpha}\right)^{-}\right](e) \\
& =\frac{1}{n^{6}} \lim _{\alpha} \sum_{\beta<b(G)}\left[\left(\tilde{u}_{k r}^{\beta} \cdot P_{\beta}\right)\left\{\left(\tilde{u}_{r 1}^{\beta} \cdot Q\right)\left(\tilde{u}_{1 l}^{\beta} \cdot P_{\beta}\right)\left(v_{i j} \overline{\tilde{u}}_{k l}^{\alpha}\right)^{\prime}\right\}\right](e) \\
& =\frac{1}{n^{6}} \lim _{\alpha} \sum_{\beta<b(G)} \int_{N_{\beta}} \tilde{u}_{k r}^{\beta}(t)\left\{\left(\tilde{u}_{r 1}^{\beta} \cdot Q\right)\left(\tilde{u}_{1 l}^{\beta} \cdot P_{\beta}\right)\left(v_{i j} \overline{\tilde{u}}_{k l}^{\alpha}\right)^{-}\right\}\left(t^{-1}\right) d \lambda_{\beta}(t) \\
& =\frac{1}{n^{6}} \lim _{\alpha} \sum_{\beta<b(G)} \int_{N_{\beta}} \tilde{u}_{k r}^{\beta}(t)\left\langle\tilde{u}_{r 1}^{\beta} \cdot Q,{ }_{t}\left[\left\{\left(\tilde{u}_{1 l}^{\beta} \cdot P_{\beta}\right)\left(v_{i j} \overline{\tilde{u}}_{k l}^{\alpha}\right)^{-}\right\}\right]\right\rangle d \lambda_{\beta}(t), \quad(16)
\end{aligned}
$$

where to obtain the fifth equation we have used Lemma 3.2. On the other hand, for every $s \in G$ we have

$$
\begin{aligned}
{ }_{t}\left[\left\{\left(\tilde{u}_{1 l}^{\beta} \cdot P_{\beta}\right)\left(v_{i j} \overline{\tilde{u}}_{k l}^{\alpha}\right)^{\prime}\right\}\right](s) & =\left\{\left(\tilde{u}_{1 l}^{\beta} \cdot P_{\beta}\right)\left(v_{i j} \overline{\tilde{u}}_{k l}^{\alpha}\right)^{\prime}\right\}\left(s^{-1} t^{-1}\right) \\
& =\int_{N_{\beta}} \tilde{u}_{1 l}^{\beta}(x)\left(v_{i j} \overline{\tilde{u}}_{k l}^{\alpha}\right)^{\gamma}\left(x^{-1} s^{-1} t^{-1}\right) d \lambda_{\beta}(x) \\
& =\int_{N_{\beta}} \tilde{u}_{1 l}^{\beta}(x)\left(v_{i j} \overline{\tilde{u}}_{k l}^{\alpha}\right)(t s x) d \lambda_{\beta}(x) \\
& =\int_{N_{\beta}} \tilde{u}_{1 l}^{\beta}(x)_{t}\left(v_{i j} \overline{\tilde{u}}_{k l}^{\alpha}\right)_{x}(s) d \lambda_{\beta}(x) .
\end{aligned}
$$


In turn, writing $\widetilde{U}_{\alpha}=\left(\tilde{u}_{l k}^{\alpha}\right)_{1 \leq l, k \leq n^{\prime}}$ and using (14),

$$
{ }_{t}\left(v_{i j} \overline{\tilde{u}}_{k l}^{\alpha}\right)_{x}=\sum_{p=1}^{d} \sum_{q=1}^{n^{\prime}} \sum_{p^{\prime}=1}^{d} \sum_{q^{\prime}=1}^{n^{\prime}} \overline{\tilde{u}}_{k q}^{\alpha}(t) \overline{\tilde{u}}_{q q^{\prime}}^{\alpha} \overline{\tilde{u}}_{q^{\prime} l}^{\alpha}(x) v_{i p}(t) v_{p p^{\prime}} v_{p^{\prime} j}(x) .
$$

Accordingly, by (17) and (18)

$$
\begin{aligned}
& t\left[\left\{\left(\tilde{u}_{1 l}^{\beta} \cdot P_{\beta}\right)\left(v_{i j} \overline{\tilde{u}}_{k l}^{\alpha}\right)^{\prime}\right\}\right](s)= \\
& \quad \sum_{p=1}^{d} \sum_{q=1}^{n^{\prime}} \sum_{p^{\prime}=1}^{d} \sum_{q^{\prime}=1}^{n^{\prime}} \overline{\tilde{u}}_{k q}^{\alpha}(t) \overline{\tilde{u}}_{q q^{\prime}}^{\alpha}(s) v_{i p}(t) v_{p p^{\prime}}(s) \int_{N_{\beta}} \tilde{u}_{1 l}^{\beta}(x) \overline{\tilde{u}}_{q^{\prime} l}^{\alpha}(x) v_{p^{\prime} j}(x) d \lambda_{\beta}(x) .
\end{aligned}
$$

Therefore, by (16) and Lemma 3.4 we have

$$
\begin{aligned}
\left\langle C_{k l} \cdot P_{k l r}, v_{i j}\right\rangle=\frac{1}{n^{6}} \lim _{\alpha} \sum_{p=1}^{d} \sum_{q=1}^{n^{\prime}} \sum_{p^{\prime}=1}^{d} \sum_{q^{\prime}=1}^{n^{\prime}} \sum_{\beta<b(G)}\left\langle\tilde{u}_{r 1}^{\beta} \cdot Q, \overline{\tilde{u}}_{q q^{\prime}}^{\alpha} v_{p p^{\prime}}\right\rangle \\
\quad \int_{N_{\beta}} \tilde{u}_{k r}^{\beta}(t) \overline{\tilde{u}}_{k q}^{\alpha}(t) v_{i p}(t) d \lambda_{\beta}(t) \int_{N_{\beta}} \tilde{u}_{1 l}^{\beta}(x) \overline{\tilde{u}}_{q^{\prime} l}^{\alpha}(x) v_{p^{\prime} j}(x) d \lambda_{\beta}(x) \\
=\frac{1}{n^{6}} \lim _{\alpha} \sum_{p=1}^{d} \sum_{q=1}^{n} \sum_{p^{\prime}=1}^{d} \sum_{q^{\prime}=1}^{n}\left\langle\tilde{u}_{r 1}^{\alpha} \cdot Q, \overline{\tilde{u}}_{q q^{\prime}}^{\alpha} v_{p p^{\prime}}\right\rangle \\
\quad \int_{N_{\alpha}} \tilde{u}_{k r}^{\alpha}(t) \overline{\tilde{u}}_{k q}^{\alpha}(t) v_{i p}(t) d \lambda_{\alpha}(t) \int_{N_{\alpha}} \tilde{u}_{1 l}^{\alpha}(x) \overline{\tilde{u}}_{q^{\prime} l}^{\alpha}(x) v_{p^{\prime} j}(x) d \lambda_{\alpha}(x),
\end{aligned}
$$

in the last equation the summations over $q$ and $q^{\prime}$ were carried up to $n$ since by $(4), \overline{\tilde{u}}_{k q}^{\alpha}(t)=\overline{\tilde{u}}_{q^{\prime} l}^{\alpha}(x)=0$ if $t, x \in N_{\alpha}$, and $q, q^{\prime}>n$. Hence

$$
\begin{aligned}
& \sum_{k=1}^{n} \sum_{l=1}^{n}\left\langle C_{k l} \cdot P_{k l r}, v_{i j}\right\rangle= \\
& \frac{1}{n^{6}} \lim _{\alpha} \sum_{p=1}^{d} \sum_{q=1}^{n} \sum_{p^{\prime}=1}^{d} \sum_{q^{\prime}=1}^{n} \int_{N_{\alpha}} \sum_{k=1}^{n} \tilde{u}_{k r}^{\alpha}(t) \overline{\tilde{u}}_{k q}^{\alpha}(t) v_{i p}(t) d \lambda_{\alpha}(t)\left\langle\tilde{u}_{r 1}^{\alpha} \cdot Q, \overline{\tilde{u}}_{q q^{\prime}}^{\alpha} v_{p p^{\prime}}\right\rangle \\
& \int_{N_{\alpha}} \sum_{l=1}^{n} \tilde{u}_{1 l}^{\alpha}(x) \overline{\tilde{u}}_{q^{\prime} l}^{\alpha}(x) v_{p^{\prime} j}(x) d \lambda_{\alpha}(x) \\
& =\frac{1}{n^{6}} \lim _{\alpha} \sum_{p=1}^{d} \sum_{q=1}^{n} \sum_{p^{\prime}=1}^{d} \sum_{q^{\prime}=1}^{n} \int_{N_{\alpha}} n^{2} \delta_{r q} v_{i p}(t) d \lambda_{\alpha}(t)\left\langle\tilde{u}_{r 1}^{\alpha} \cdot Q, \overline{\tilde{u}}_{q q^{\prime}}^{\alpha} v_{p p^{\prime}}\right\rangle \\
& \int_{N_{\alpha}} n^{2} \delta_{1 q^{\prime}} v_{p^{\prime} j}(x) d \lambda_{\alpha}(x) \\
& =\frac{1}{n^{2}} \lim _{\alpha} \sum_{p=1}^{d} \sum_{p^{\prime}=1}^{d} \int_{N_{\alpha}} v_{i p}(t) d \lambda_{\alpha}(t)\left\langle Q, \tilde{u}_{r 1}^{\alpha} \overline{\tilde{u}}_{r 1}^{\alpha} v_{p p^{\prime}}\right\rangle \int_{N_{\alpha}} v_{p^{\prime} j}(x) d \lambda_{\alpha}(x) .
\end{aligned}
$$


Finally, summing over $r$ leads to

$$
\begin{aligned}
& \sum_{r=1}^{n^{\prime}} \sum_{k=1}^{n} \sum_{l=1}^{n}\left\langle C_{k l} \cdot P_{k l r}, v_{i j}\right\rangle \\
& =\frac{1}{n^{2}} \lim _{\alpha} \sum_{p=1}^{d} \sum_{p^{\prime}=1}^{d} \int_{N_{\alpha}} v_{i p}(t) d \lambda_{\alpha}(t)\left\langle Q, \sum_{r=1}^{n^{\prime}} \tilde{u}_{r 1}^{\alpha} \overline{\tilde{u}}_{r 1}^{\alpha} v_{p p^{\prime}}\right\rangle \int_{N_{\alpha}} v_{p^{\prime} j}(x) d \lambda_{\alpha}(x) \\
& =\frac{1}{n^{2}} \lim _{\alpha} \sum_{p=1}^{d} \sum_{p^{\prime}=1}^{d} \int_{N_{\alpha}} v_{i p}(t) d \lambda_{\alpha}(t)\left\langle Q, n^{2} v_{p p^{\prime}}\right\rangle \int_{N_{\alpha}} v_{p^{\prime} j}(x) d \lambda_{\alpha}(x) \\
& =\lim _{\alpha} \sum_{p=1}^{d} \sum_{p^{\prime}=1}^{d} \int_{N_{\alpha}} v_{i p}(t) d \lambda_{\alpha}(t)\left\langle Q, v_{p p^{\prime}}\right\rangle \int_{N_{\alpha}} v_{p^{\prime} j}(x) d \lambda_{\alpha}(x) \\
& =\sum_{p=1}^{d} \sum_{p^{\prime}=1}^{d} v_{i p}(e)\left\langle Q, v_{p p^{\prime}}\right\rangle v_{p^{\prime} j}(e) \\
& =\left\langle Q, v_{i j}\right\rangle
\end{aligned}
$$

Thus,

$$
Q=\sum_{r=1}^{n^{\prime}} \sum_{k=1}^{n} \sum_{l=1}^{n} C_{k l} \cdot P_{k l r}=\sum_{k=1}^{n} \sum_{l=1}^{n} C_{k l} \cdot P_{k l} .
$$

The following definition generalizes the concept of factorization property of level $\kappa$ introduced by Neufang in [45].

Definition 3.10. Let $A$ be a Banach algebra, and let $\kappa$ be a cardinal number. We say that $A^{*}$ has the weak left $A^{* *}$ factorization property of level $\kappa$ [property $\left.\left(W F_{\kappa}\right)\right]$ if for each family of functionals $\left\{h_{\alpha}: \alpha \in\right.$ $I\} \subset \operatorname{Ball}\left(A^{*}\right)$ with $|I|=\kappa$, there exists a family $\left\{\Psi_{\alpha}^{i}: \alpha \in I, i=\right.$ $1, \ldots, m\} \subset \operatorname{Ball}\left(A^{* *}\right)$ and a family of functionals $\left\{h_{i}: i=1, \ldots, m\right\}$ in $A^{*}$ such that

$$
h_{\alpha}=\sum_{i=1}^{m} \Psi_{\alpha}^{i} \cdot h_{i} \quad(\alpha \in I) .
$$

If $\Psi_{\alpha}^{i}$ can be chosen independent of $h_{\alpha}$, then we say that $A^{*}$ has the weak left uniform $A^{* *}$ factorization property of level $\kappa$ [property $\left.\left(W U F_{\kappa}\right)\right]$.

Theorem 3.11. Let $G$ be a compact group such that $b(G)$ has uncountable cofinality. Then $A(G)^{*}=V N(G)$ has property $\left(W U F_{b(G)}\right)$. In other words, there exists a family $\left\{C_{k l}^{\lambda}: \lambda<b(G), 1 \leq k, l \leq n\right\}$ of functionals in $A(G)^{* *}$ such that for every bounded family $\left\{Q_{\lambda}: \lambda<b(G)\right\}$ of operators in $V N(G)$, there exists a family of operators $\left\{\widehat{P}_{k l}: 1 \leq\right.$ 
$k, l \leq n\}$ in $V N(G)$ with

$$
Q_{\lambda}=\sum_{k=1}^{n} \sum_{l=1}^{n} C_{k l}^{\lambda} \cdot \widehat{P}_{k l} \quad(\lambda<b(G)) .
$$

Proof. We partition $b(G)$ into $b(G)$ subsets $I_{\lambda}$ such that each $I_{\lambda}$ is cofinal in $b(G)$ (see [49, Lemma, p. 61]). To avoid confusion, we use $\lambda, \gamma, \ldots$ to index the sets $I_{\lambda}$ partitioning $b(G)$, and we write $\alpha \in I_{\lambda}$, $\beta \in I_{\gamma}, \ldots$, for elements of these sets. Note that the cofinality of $I_{\lambda}$ ensures that $\cap\left\{N_{\alpha}: \alpha \in I_{\lambda}\right\}=\{e\}$ (this is crucial in the proof of Theorem 3.9). For each $\lambda<b(G)$ and each $1 \leq k, l \leq n$, let $C_{k l}^{\lambda}$ be a cluster point of $\left\{\overline{\tilde{u}}_{k l}^{\alpha}: \alpha \in I_{\lambda}\right\}$ in $A(G)^{* *}$. As in Lemma 3.8 we define:

$$
\begin{aligned}
P_{k l r}^{\gamma} & =\frac{1}{n^{6}} \sum_{\beta \in I_{\gamma}}\left(\tilde{u}_{k r}^{\beta} \cdot P_{\beta}\right)\left(\tilde{u}_{r 1}^{\beta} \cdot Q_{\gamma}\right)\left(\tilde{u}_{1 l}^{\beta} \cdot P_{\beta}\right) \\
& =\frac{1}{n^{6}} \sum_{\beta \in I_{\gamma}}\left(\tilde{u}_{k k}^{\beta} \cdot P_{\beta}\right) T_{\gamma}^{\beta}\left(\tilde{u}_{l l}^{\beta} \cdot P_{\beta}\right) \quad\left(w^{*} \text {-limits }\right),
\end{aligned}
$$

where

$$
T_{\gamma}^{\beta}=\left(\tilde{u}_{k r}^{\beta} \cdot P_{\beta}\right)\left(\tilde{u}_{r 1}^{\beta} \cdot Q_{\gamma}\right)\left(\tilde{u}_{1 l}^{\beta} \cdot P_{\beta}\right) .
$$

Thus each $P_{k l r}^{\gamma}$ is defined so that if we set $P_{k l}^{\gamma}=\sum_{r=1}^{n^{\prime}} P_{k l r}^{\gamma}$, then upon replacing $Q$ with $Q_{\gamma}$ and $P_{k l}$ with $P_{k l}^{\gamma}$, the factorization result in Theorem 3.9 holds for $Q_{\gamma}$, that is,

$$
Q_{\gamma}=\sum_{k=1}^{n} \sum_{l=1}^{n} C_{k l}^{\gamma} \cdot P_{k l}^{\gamma} \quad \text { for each } \gamma<b(G) .
$$

Next we define

$$
\widehat{P}_{k l r}=\sum_{\gamma<b(G)} P_{k l r}^{\gamma} \quad\left(w^{*} \text {-limit }\right) .
$$

The convergence of the sum follows from Lemma 3.7 once we substitute for $P_{k l r}^{\gamma}$ from (24). We claim that for each $1 \leq k, l \leq n$ and each $\lambda<b(G)$ we have

$$
C_{k l}^{\lambda} \cdot \widehat{P}_{k l r}=C_{k l}^{\lambda} \cdot P_{k l r}^{\lambda}
$$

In fact, if $v \in A(G)$ is such that $v(x)=v_{i j}(x)=\left(V(x) e_{j} \mid e_{i}\right)$ where $V \in \widehat{G}, \operatorname{dim} V=d$, and $\left\{e_{1}, \ldots, e_{d}\right\}$ is the standard basis of $\mathbf{C}^{d}$, then as we obtained in the equation preceding to (19), we can write

$$
\begin{aligned}
\left\langle C_{k l}^{\lambda} \cdot \widehat{P}_{k l r}, v_{i j}\right\rangle=\lim _{\alpha \in I_{\lambda}}\left\langle\overline{\tilde{u}}_{k l}^{\alpha} \cdot \sum_{\gamma<b(G)} P_{k l r}^{\gamma}, v_{i j}\right\rangle=\lim _{\alpha \in I_{\lambda}} \sum_{\gamma<b(G)}\left\langle\overline{\tilde{u}}_{k l}^{\alpha} \cdot P_{k l r}^{\gamma}, v_{i j}\right\rangle \\
=\frac{1}{n^{6}} \lim _{\alpha \in I_{\lambda}} \sum_{\gamma<b(G)} \sum_{p=1}^{d} \sum_{q=1}^{n^{\prime}} \sum_{p^{\prime}=1}^{d} \sum_{q^{\prime}=1}^{n^{\prime}} \sum_{\beta \in I_{\gamma}}\left\langle\tilde{u}_{r 1}^{\beta} \cdot Q_{\gamma}, \overline{\tilde{u}}_{q q^{\prime}}^{\alpha} v_{p p^{\prime}}\right\rangle \\
\int_{N_{\beta}} \tilde{u}_{k r}^{\beta}(t) \overline{\tilde{u}}_{k q}^{\alpha}(t) v_{i p}(t) d \lambda_{\beta}(t) \int_{N_{\beta}} \tilde{u}_{1 l}^{\beta}(x) \overline{\tilde{u}}_{q^{\prime} l}^{\alpha}(x) v_{p^{\prime} j}(x) d \lambda_{\beta}(x),
\end{aligned}
$$


where by Lemma 3.4, for $\alpha \in I_{\lambda}$ large enough, if $\beta \in I_{\gamma}$ and $\gamma \neq \lambda$, we have

$$
\int_{N_{\beta}} \tilde{u}_{1 l}^{\beta}(x) \overline{\tilde{u}}_{q^{\prime} l}^{\alpha}(x) v_{p^{\prime} j}(x) d \lambda_{\beta}(x)=0 .
$$

Thus repeating the calculations that led to (19) backward, we can write

$$
\lim _{\alpha \in I_{\lambda}}\left\langle\sum_{\gamma<b(G)} \overline{\tilde{u}}_{k l}^{\alpha} \cdot P_{k l r}^{\gamma}, v_{i j}\right\rangle=\lim _{\alpha \in I_{\lambda}}\left\langle\overline{\tilde{u}}_{k l}^{\alpha} \cdot P_{k l r}^{\lambda}, v_{i j}\right\rangle=\left\langle C_{k l}^{\lambda} \cdot P_{k l r}^{\lambda}, v_{i j}\right\rangle,
$$

which proves (26). Using (26), for each $\lambda<b(G)$, we have

$$
\sum_{r=1}^{n^{\prime}} \sum_{k=1}^{n} \sum_{l=1}^{n} C_{k l}^{\lambda} \cdot \widehat{P}_{k l r}=\sum_{r=1}^{n^{\prime}} \sum_{k=1}^{n} \sum_{l=1}^{n} C_{k l}^{\lambda} \cdot P_{k l r}^{\lambda}=Q_{\lambda} .
$$

Now our result follows easily from the preceding identity if we define $\widehat{P}_{k l}=\sum_{r=1}^{n^{\prime}} \widehat{P}_{k r l}$.

In order to apply our factorization Theorem 3.11 to the problem of determining the centre of $A(G)^{* *}$ we recall the following definition $([45])$.

Definition 3.12. Let $X$ be a Banach space and $\kappa \geq \aleph_{0}$ a cardinal number.

(i) A functional $f \in X^{* *}$ is called $w^{*}-\kappa$-continuous if for all nets $\left(x_{\alpha}\right)_{\alpha} \subset \operatorname{Ball}\left(X^{*}\right)$ of cardinality $\aleph_{0} \leq|I| \leq \kappa$ with $x_{\alpha} \rightarrow 0$ in $w^{*}$-topology, we have $f\left(x_{\alpha}\right) \rightarrow 0$.

(ii) We say that $X$ has the Mazur property of level $\kappa$ [property $\left(M_{\kappa}\right)$ ] if every $w^{*}-\kappa$-continuous functional $f \in X^{* *}$ is an element of $X$.

Our next theorem is a generalization of two results by Neufang (see [45, Theorem 2.3 and Theorem 4.2]). In our theorem, the factorization property of level $\kappa$ has been replaced with the weak factorization property of level $\kappa$. The theorem can be proved by straightforward modifications of the original proofs. We recall that given a Banach algebra $A$, the right multiplier algebra of $A$ is defined by

$$
R M(A)=\{T \in \mathcal{B}(A): T(a b)=a T(b) \text { for all } a, b \in A\},
$$

where $\mathcal{B}(A)$ is the Banach algebra of all bounded operators on $A$.

Theorem 3.13. Let $A$ be a Banach algebra satisfying $\left(M_{\kappa}\right)$ and whose dual $A^{*}$ has the property $\left(W F_{\kappa}\right)$, for some $\kappa \geq \aleph_{0}$. Then

(i) $A$ is left strongly Arens irregular; that is, $\mathfrak{Z}_{t}^{(l)}\left(A^{* *}\right)=A$;

(ii) every left $A^{* *}$-module homomorphism on $A^{*}$ is automatically bounded and $w^{*}-w^{*}$-continuous; that is,

$$
\operatorname{Hom}_{A^{* *}}\left(A^{*}\right)=\mathcal{B}_{A^{* *}}^{\sigma}\left(A^{*}\right) ;
$$

(iii) if $A$ has a right approximate identity bounded by 1 , then the left topological centre of $\left(A^{*} \cdot A\right)^{*}$ is isometrically isomorphic to the algebra $R M(A)$, that is, $\mathfrak{Z}_{t}^{(l)}\left(\left(A^{*} \cdot A\right)^{*}\right) \cong R M(A)$. 
Concerning the above theorem we remark that assuming the right version of the weak factorization property $\left(W F_{\kappa}\right)$, one can deduce the right strong Arens irregularity of $A$ and the continuity of right $A^{* *}$ module maps on $A^{*}$.

It is shown by Hu-Neufang [28, Corollary 4.39(i)] that for all locally compact groups $G, A(G)$ has the Mazur property of level $b(G) \cdot \aleph_{0}$. It is also known that for amenable groups the Fourier algebra $A(G)$ has an approximate identity bounded by 1 . These results together with our Theorems 3.11 and 3.13 imply the following corollary.

Corollary 3.14. Let $G$ be a compact group such that $b(G)$ has uncountable cofinality. Then

(i) $A(G)$ is strongly Arens irregular;

(ii) every left (right) $A(G)^{* *}$-module homomorphism on $A(G)^{*}$ is automatically bounded and $w^{*}-w^{*}$-continuous;

(iii) $\mathfrak{Z}_{t}\left(U C_{2}(G)^{*}\right) \cong B(G)$.

For statement (iii) we note that since $G$ is amenable, $R M(A(G))=$ $B(G)$ by Derighetti [7, Theorem 9$]$. We should also point out that by Lau-Losert [37, Theorem 6.4], for all amenable groups the statement (iii) implies (i).

Remark 3.15. F. Ghahramani and, independently, Hofmeier-Wittstock [23] asked the question whether a left $L^{\infty}(G)^{*}$-module homomorphism on $L^{\infty}(G)$ is automatically bounded and, hence, normal (that is, $w^{*}-w^{*}$ continuous, see Ghahramani-McClure [17]). This question (in a stronger form) was answered affirmatively for all non-compact locally compact groups by Neufang in [43, Theorem 3.1]. Our result in Corollary 3.14(ii) shows that the dual version of the Ghahramani-Hofmeier-Wittstock question, in which $L^{\infty}(G)$ is replaced by $V N(G)$, has an affirmative answer if $G$ is a compact group whose local weight has uncountable cofinality (see also Corollary 4.7).

\section{A Factorization Theorem for $V N(G \times H)$}

Throughout this section, we assume that $G$ is a compact group whose local weight has uncountable cofinality, and that $H$ is an amenable locally compact group. Using the results in the previous section, we shall prove a factorization theorem for $V N(G \times H)$ and apply this result to prove the strong Arens irregularity of $A(G \times H)$. We shall continue to use the notation introduced in Section 3. If $u \in B(G)$, we define $\mathfrak{u} \in B(G \times H)$ to be the function defined by $\mathfrak{u}(s, t)=u(s)$; that is, $\mathfrak{u}=u \otimes 1_{H}$, where $1_{H}$ is the function with the constant value 1 on $H$. Also if $T \in V N(G)$, we define $\widetilde{T}=T \otimes$ I where I is the identity operator on $L^{2}(H)$; therefore, $\widetilde{T} \in V N(G \times H)$ and $\widetilde{T}(f \otimes h)=T f \otimes h$ $\left(f \in L^{2}(G), h \in L^{2}(H)\right)$. Note that in view of the above conventions, we have $\tilde{\mathfrak{u}}_{i j}^{\alpha}=\tilde{u}_{i j}^{\alpha} \otimes 1_{H} \in B(G \times H)$ and $\widetilde{P}_{\alpha}=P_{\alpha} \otimes \mathrm{I} \in V N(G \times H)$. 
The proof of the following lemma is straightforward and is omitted.

Lemma 4.1. Let $G_{i}$ be a locally compact group, $u_{i} \in B\left(G_{i}\right), T_{i} \in$ $V N\left(G_{i}\right)(i=1,2)$. Then,

$$
\left(u_{1} \otimes u_{2}\right) \cdot\left(T_{1} \otimes T_{2}\right)=\left(u_{1} \cdot T_{1}\right) \otimes\left(u_{2} \cdot T_{2}\right) .
$$

Lemma 4.2. The operators $\tilde{\mathfrak{u}}_{i j}^{\alpha} \cdot \widetilde{P}_{\alpha}$ in $V N(G \times H)$ satisfy orthogonality relations

$$
\left(\tilde{\mathfrak{u}}_{i j}^{\alpha} \cdot \widetilde{P}_{\alpha}\right)\left(\tilde{\mathfrak{u}}_{k l}^{\beta} \cdot \widetilde{P}_{\beta}\right)=\delta_{\alpha \beta} \delta_{j k}\left(\tilde{\mathfrak{u}}_{i l}^{\alpha} \cdot \widetilde{P}_{\alpha}\right)
$$

whenever $1 \leq i, j, k \leq n$ and $1 \leq l \leq n^{\prime}$.

Proof. Using Lemma 4.1 and Lemma 3.5 we can write

$$
\begin{aligned}
\left(\tilde{\mathfrak{u}}_{i j}^{\alpha} \cdot \widetilde{P}_{\alpha}\right)\left(\tilde{\mathfrak{u}}_{k l}^{\beta} \cdot \widetilde{P}_{\beta}\right) & =\left(\tilde{u}_{i j}^{\alpha} \cdot P_{\alpha} \otimes \mathrm{I}\right)\left(\tilde{u}_{k l}^{\beta} \cdot P_{\beta} \otimes \mathrm{I}\right) \\
& =\left(\tilde{u}_{i j}^{\alpha} \cdot P_{\alpha}\right)\left(\tilde{u}_{k l}^{\beta} \cdot P_{\beta}\right) \otimes \mathrm{I} \\
& =\delta_{\alpha \beta} \delta_{j k}\left(\tilde{u}_{i l}^{\alpha} \cdot P_{\alpha}\right) \otimes \mathrm{I} \\
& =\delta_{\alpha \beta} \delta_{j k}\left(\tilde{\mathfrak{u}}_{i l}^{\alpha} \cdot \widetilde{P}_{\alpha}\right) .
\end{aligned}
$$

Lemma 4.3. For every $\alpha<b(G)$ and $1 \leq k, l \leq n$, $\left(\tilde{\mathfrak{u}}_{k l}^{\alpha} \cdot \widetilde{P}_{\alpha}\right)^{*}=\tilde{\mathfrak{u}}_{l k}^{\alpha} \cdot \widetilde{P}_{\alpha}$. In particular, $\tilde{\mathfrak{u}}_{k k}^{\alpha} \cdot \widetilde{P}_{\alpha}$ is an orthogonal projection.

Proof. By Lemma 4.1 and Lemma 3.6 we have

$$
\left(\tilde{\mathfrak{u}}_{k l}^{\alpha} \cdot \widetilde{P}_{\alpha}\right)^{*}=\left(\tilde{u}_{k l}^{\alpha} \cdot P_{\alpha}\right)^{*} \otimes \mathrm{I}=\left(\tilde{u}_{l k}^{\alpha} \cdot P_{\alpha}\right) \otimes \mathrm{I}=\tilde{\mathfrak{u}}_{l k}^{\alpha} \cdot \widetilde{P}_{\alpha} .
$$

Of course, by Lemma 4.2 we have $\left(\tilde{\mathfrak{u}}_{k k}^{\alpha} \cdot \widetilde{P}_{\alpha}\right)^{2}=\tilde{\mathfrak{u}}_{k k}^{\alpha} \cdot \widetilde{P}_{\alpha}$, proving that it is an orthogonal projection.

The proof of our next lemma is similar to that of Lemma 3.8. We omit the proof for briefness.

Lemma 4.4. Let $\mathcal{Q} \in V N(G \times H)$ and let $1 \leq k, l \leq n$ and $1 \leq r \leq n^{\prime}$. Then the sum

$$
\mathcal{P}_{k l r}=\frac{1}{n^{6}} \sum_{\beta<b(G)}\left(\tilde{\mathfrak{u}}_{k r}^{\beta} \cdot \widetilde{P}_{\beta}\right)\left(\tilde{\mathfrak{u}}_{r 1}^{\beta} \cdot \mathcal{Q}\right)\left(\tilde{\mathfrak{u}}_{1 l}^{\beta} \cdot \widetilde{P}_{\beta}\right)
$$

is $w^{*}$-convergent in $V N(G \times H)$.

Lemma 4.5. Let $1 \leq k, l \leq n$ and $1 \leq r \leq n^{\prime}$. Let $\xi_{k l}$ be a $w^{*}$ cluster point of the net $\left(\overline{\tilde{\mathfrak{u}}}_{k l}^{\alpha}\right)_{\alpha<b(G)}$ in $U C_{2}(G \times H)^{*}$. Then, for every $\mathcal{Q} \in V N(G \times H)$, we have

$$
\mathcal{Q}=\sum_{k=1}^{n} \sum_{l=1}^{n} \xi_{k l} \odot \mathcal{P}_{k l}=\sum_{k=1}^{n} \sum_{l=1}^{n} \widetilde{\xi}_{k l} \cdot \mathcal{P}_{k l}
$$

where $\mathcal{P}_{k l}=\sum_{r=1}^{n^{\prime}} \mathcal{P}_{k l r}$ and $\widetilde{\xi}_{k l}$ is any Hahn-Banach extension of $\xi_{k l}$ to an element in $\operatorname{VN}(G \times H)^{*}$. 
Proof. Since $G \times H$ is amenable it follows from Lau-Losert [37, Propisition 4.3] that $B(G \times H)$ embeds canonically into $U C_{2}(G \times H)^{*}$. Since the net $\left(\overline{\tilde{\mathfrak{u}}}_{k l}^{\alpha}\right)_{\alpha<b(G)}$ is bounded it has a $w^{*}$-cluster point, and indeed it is possible to assume without loss of generality that $\overline{\tilde{\mathfrak{u}}}_{k l}^{\alpha} \rightarrow \xi_{k l}$ in the $w^{*}$-topology of $U C_{2}(G \times H)^{*}$.

Let $v \in A(G)$ and $w \in A(H)$. As in the proof of Theorem 3.9 we may assume that for $s \in G, v(s)=v_{i j}(s)=\left(V(s) e_{j} \mid e_{i}\right)$, where $V \in \widehat{G}$, $\operatorname{dim} V=d$, and $\left\{e_{1}, \ldots, e_{d}\right\}$ is the standard basis of $\mathbf{C}^{d}$. Suppose first that $\mathcal{Q}=Q_{1} \otimes Q_{2}$. We can write

$$
\begin{aligned}
& \left\langle\xi_{k l} \odot \mathcal{P}_{k l r}, v_{i j} \otimes w\right\rangle=\left\langle\xi_{k l}, \mathcal{P}_{k l r} \cdot\left(v_{i j} \otimes w\right)\right\rangle \\
& \quad=\lim _{\alpha}\left\langle\frac{1}{n^{6}} \sum_{\beta<b(G)}\left(\tilde{\mathfrak{u}}_{k r}^{\beta} \cdot \widetilde{P}_{\beta}\right)\left(\tilde{\mathfrak{u}}_{r 1}^{\beta} \cdot \mathcal{Q}\right)\left(\tilde{\mathfrak{u}}_{1 l}^{\beta} \cdot \widetilde{P}_{\beta}\right),\left(v_{i j} \otimes w\right) \overline{\tilde{\mathfrak{u}}}_{k l}^{\alpha}\right\rangle .
\end{aligned}
$$

But

$$
\begin{aligned}
& \left\langle\left(\tilde{\mathfrak{u}}_{k r}^{\beta} \cdot \widetilde{P}_{\beta}\right)\left(\tilde{\mathfrak{u}}_{r 1}^{\beta} \cdot \mathcal{Q}\right)\left(\tilde{\mathfrak{u}}_{1 l}^{\beta} \cdot \widetilde{P}_{\beta}\right),\left(v_{i j} \otimes w\right) \overline{\tilde{\mathfrak{u}}}_{k l}^{\alpha}\right\rangle \\
& =\left\langle\left(\left(\tilde{u}_{k r}^{\beta} \cdot P_{\beta}\right) \otimes \mathrm{I}\right)\left(\left(\tilde{u}_{r 1}^{\beta} \cdot Q_{1}\right) \otimes Q_{2}\right)\left(\left(\tilde{u}_{1 l}^{\beta} \cdot P_{\beta}\right) \otimes \mathrm{I}\right), v_{i j} \overline{\tilde{u}}_{k l}^{\alpha} \otimes w\right\rangle \\
& =\left\langle\left(\tilde{u}_{k r}^{\beta} \cdot P_{\beta}\right)\left(\tilde{u}_{r 1}^{\beta} \cdot Q_{1}\right)\left(\tilde{u}_{1 l}^{\beta} \cdot P_{\beta}\right), v_{i j} \overline{\tilde{u}}_{k l}^{\alpha}\right\rangle\left\langle Q_{2}, w\right\rangle .
\end{aligned}
$$

Then repeating the calculations that led to (15) and subsequently to (22), we can write

$$
\begin{aligned}
& \sum_{r=1}^{n^{\prime}} \sum_{k=1}^{n} \sum_{l=1}^{n}\left\langle\xi_{k l} \odot \mathcal{P}_{k l r}, v_{i j} \otimes w\right\rangle \\
& =\frac{1}{n^{6}} \lim _{\alpha} \sum_{r=1}^{n^{\prime}} \sum_{k=1}^{n} \sum_{l=1}^{n} \sum_{\beta<b(G)}\left\langle\left(\tilde{u}_{k r}^{\beta} \cdot P_{\beta}\right)\left(\tilde{u}_{r 1}^{\beta} \cdot Q_{1}\right)\left(\tilde{u}_{1 l}^{\beta} \cdot P_{\beta}\right), v_{i j} \overline{\tilde{u}}_{k l}^{\alpha}\right\rangle\left\langle Q_{2}, w\right\rangle \\
& =\left\langle Q_{1}, v_{i j}\right\rangle\left\langle Q_{2}, w\right\rangle=\left\langle Q_{1} \otimes Q_{2}, v_{i j} \otimes w\right\rangle .
\end{aligned}
$$

Now let $\mathcal{Q} \in V N(G \times H)$ be arbitrary. Since by Effros-Ruan [9],

$$
V N(G) \bar{\otimes} V N(H) \cong\left(A(G) \widehat{\otimes}_{\mathrm{op}} A(H)\right)^{*} \cong A(G \times H)^{*}=V N(G \times H),
$$


we can pick a net $\left(\mathcal{Q}_{F}\right)$ such that $\mathcal{Q}_{F}=\sum_{i \in F} Q_{1}^{i} \otimes Q_{2}^{i}$ for some finite subset $F$ of $\mathbf{N}$ and $\mathcal{Q}$ is the $w^{*}$-limit of $\left(\mathcal{Q}_{F}\right)$. Then

$$
\begin{aligned}
\langle & \left.\xi_{k l} \odot \mathcal{P}_{k l r}, v_{i j} \otimes w\right\rangle \\
= & \frac{1}{n^{6}} \lim _{\alpha} \sum_{\beta<b(G)}\left\langle\left(\tilde{\mathfrak{u}}_{k r}^{\beta} \cdot \widetilde{P}_{\beta}\right)\left(\tilde{\mathfrak{u}}_{r 1}^{\beta} \cdot \mathcal{Q}\right)\left(\tilde{\mathfrak{u}}_{1 l}^{\beta} \cdot \widetilde{P}_{\beta}\right),\left(v_{i j} \otimes w\right) \overline{\tilde{u}}_{k l}^{\alpha}\right\rangle \\
= & \frac{1}{n^{6}} \lim _{\alpha} \sum_{\beta<b(G)} \lim _{F} \sum_{i \in F}\left\langle\left(\tilde{\mathfrak{u}}_{k r}^{\beta} \cdot \widetilde{P}_{\beta}\right)\left(\tilde{\mathfrak{u}}_{r 1}^{\beta} \cdot\left(Q_{1}^{i} \otimes Q_{2}^{i}\right)\right)\left(\tilde{\mathfrak{u}}_{1 l}^{\beta} \cdot \widetilde{P}_{\beta}\right),\left(v_{i j} \otimes w\right) \overline{\tilde{u}}_{k l}^{\alpha}\right\rangle \\
= & \frac{1}{n^{6}} \lim _{\alpha} \sum_{\beta<b(G)} \lim _{F} \sum_{i \in F}\left\langle\left(\tilde{u}_{k r}^{\beta} \cdot P_{\beta}\right)\left(\tilde{u}_{r 1}^{\beta} \cdot Q_{1}^{i}\right)\left(\tilde{u}_{1 l}^{\beta} \cdot P_{\beta}\right), v_{i j} \overline{\tilde{u}}_{k l}^{\alpha}\right\rangle\left\langle Q_{2}^{i}, w\right\rangle \\
= & \frac{1}{n^{6}} \lim _{\alpha} \sum_{p=1}^{d} \sum_{q=1}^{n^{\prime}} \sum_{p^{\prime}=1}^{d} \sum_{q^{\prime}=1}^{n^{\prime}} \sum_{\beta<b(G)} \lim _{F} \sum_{i \in F}\left\langle\tilde{u}_{r 1}^{\beta} \cdot Q_{1}^{i}, \overline{\tilde{u}}_{q q^{\prime}}^{\alpha} v_{p p^{\prime}}\right\rangle\left\langle Q_{2}^{i}, w\right\rangle \\
& \int_{N_{\beta}} \tilde{u}_{k r}^{\beta}(t) \overline{\tilde{u}}_{k q}^{\alpha}(t) v_{i p}(t) d \lambda_{\beta}(t) \int_{N_{\beta}} \tilde{u}_{1 l}^{\beta}(x) \overline{\tilde{u}}_{q^{\prime} l}^{\alpha}(x) v_{p^{\prime} j}(x) d \lambda_{\beta}(x) \\
= & \frac{1}{n^{6}} \lim _{\alpha} \sum_{p=1}^{d} \sum_{q=1}^{n^{\prime}} \sum_{p^{\prime}=1}^{d} \sum_{q^{\prime}=1}^{n^{\prime}} \lim _{F} \sum_{i \in F}\left\langle\tilde{u}_{r 1}^{\alpha} \cdot Q_{1}^{i}, \overline{\tilde{u}}_{q q^{\prime}}^{\alpha} v_{p p^{\prime}}\right\rangle\left\langle Q_{2}^{i}, w\right\rangle \\
& \int_{N_{\alpha}} \tilde{u}_{k r}^{\alpha}(t) \overline{\tilde{u}}_{k q}^{\alpha}(t) v_{i p}(t) d \lambda_{\alpha}(t) \int_{N_{\alpha}} \tilde{u}_{1 l}^{\alpha}(x) \overline{\tilde{u}}_{q^{\prime} l}^{\alpha}(x) v_{p^{\prime} j}(x) d \lambda_{\alpha}(x) .
\end{aligned}
$$

As in the proof of (20) and (22), summing over $k$ and $l$ and then over $r$, we obtain:

$$
\begin{aligned}
& \sum_{r=1}^{n^{\prime}} \sum_{k=1}^{n} \sum_{l=1}^{n}\left\langle\xi_{k l} \odot \mathcal{P}_{k l r}, v_{i j} \otimes w\right\rangle \\
& =\lim _{\alpha} \sum_{p=1}^{d} \sum_{p^{\prime}=1}^{d} \lim _{F} \sum_{i \in F}\left\langle Q_{1}^{i}, v_{p p^{\prime}}\right\rangle\left\langle Q_{2}^{i}, w\right\rangle \int_{N_{\alpha}} v_{i p}(t) d \lambda_{\alpha}(t) \int_{N_{\alpha}} v_{p^{\prime} j}(x) d \lambda_{\alpha}(x) \\
& =\lim _{\alpha} \sum_{p=1}^{d} \sum_{p^{\prime}=1}^{d} \lim _{F} \sum_{i \in F}\left\langle Q_{1}^{i} \otimes Q_{2}^{i}, v_{p p^{\prime}} \otimes w\right\rangle \int_{N_{\alpha}} v_{i p}(t) d \lambda_{\alpha}(t) \int_{N_{\alpha}} v_{p^{\prime} j}(x) d \lambda_{\alpha}(x) \\
& =\lim _{\alpha} \sum_{p=1}^{d} \sum_{p^{\prime}=1}^{d}\left\langle\mathcal{Q}, v_{p p^{\prime}} \otimes w\right\rangle \int_{N_{\alpha}} v_{i p}(t) d \lambda_{\alpha}(t) \int_{N_{\alpha}} v_{p^{\prime} j}(x) d \lambda_{\alpha}(x) \\
& =\left\langle\mathcal{Q}, v_{i j} \otimes w\right\rangle,
\end{aligned}
$$

leading to the identities

$$
\mathcal{Q}=\sum_{r=1}^{n^{\prime}} \sum_{k=1}^{n} \sum_{l=1}^{n} \xi_{k l} \odot \mathcal{P}_{k l r}=\sum_{k=1}^{n} \sum_{l=1}^{n} \xi_{k l} \odot \mathcal{P}_{k l}=\sum_{k=1}^{n} \sum_{l=1}^{n} \widetilde{\xi}_{k l} \cdot \mathcal{P}_{k l} .
$$


By a similar argument to that in the previous section, Lemma 4.5 can be used to prove that $A(G \times H)^{*}$ has property $\left(W U F_{b(G)}\right)$.

Theorem 4.6. Let $G$ be a compact group whose local weight has uncountable cofinality. Let $H$ be an amenable locally compact group. Then $A(G \times H)^{*}=V N(G \times H)$ has property $\left(W U F_{b(G)}\right)$. In other words, there exists a family $\left\{\widetilde{\xi}_{k l}^{\lambda}: \lambda<b(G), 1 \leq k, l \leq n\right\}$ of functionals in $A(G \times H)^{* *}$ such that for every bounded family $\left\{\mathcal{Q}_{\lambda}: \lambda<b(G)\right\}$ of operators in $V N(G \times H)$, there exists a finite family of operators $\left\{\mathcal{P}_{k l}: 1 \leq k, l \leq n\right\}$ in $V N(G \times H)$ such that for each $\lambda<b(G)$,

$$
\mathcal{Q}_{\lambda}=\sum_{k=1}^{n} \sum_{l=1}^{n} \widetilde{\xi}_{k l}^{\lambda} \cdot \mathcal{P}_{k l} .
$$

The proof of this theorem is similar to that of Theorem 3.11 and therefore is omitted for briefness. As a consequence of Theorem 3.13 and Theorem 4.6 we obtain

Corollary 4.7. Let $G$ be a compact group whose local weight has uncountable cofinality, and let $H$ be an amenable locally compact group. Then

(i) $A(G \times H)$ is strongly Arens irregular;

(ii) every left (right) $A(G \times H)^{* *}$-module homomorphism on $A(G \times H)^{*}$ is automatically bounded and $w^{*}-w^{*}$-continuous;

(iii) $\mathfrak{Z}_{t}\left(U C_{2}(G \times H)^{*}\right)=B(G \times H)$.

\section{REFERENCES}

[1] R. Arens, The adjoint of a bilinear operation, Proc. Amer. Math. Soc. 2 (1951), 839-848.

[2] M. Bożejko, Remark on Herz-Schur multipliers on free groups, Math. Ann. 258 (1981), 11-15.

[3] P. Civin and B. Yood, The second conjugate space of a Banach algebra as an algebra, Pacific J. Math. 11 (1961), 847-870.

[4] M. Cowling and U. Haagerup, Completely bounded multipliers of the Fourier algebra of a simple Lie group of real rank one, Invent. Math. 96 (1989), 507549.

[5] H. G. Dales and A. T.-M. Lau, The second duals of Beurling algebras, Memoires Amer. Math. Soc. 177(836) (2005).

[6] J. De Canniere and U. Haagerup, Multipliers of the Fourier algebras of some simple Lie groups and their discrete subgroups, Amer. J. Math. 107 (1985), $455-500$.

[7] A. Derighetti, Some results on the Fourier-Stieltjes algebra of a locally compact group, Comment. Math. Helv. 45 (1970), 219-228.

[8] P. Eymard, L'algèbre de Fourier d'un groupe localement compact, Bull. Soc. Math. France 92 (1964), 181-236.

[9] E. G. Effros and Z-J. Ruan, On approximation properties for operator spaces, Internat. J. Math. 1 (1990), 163-187.

[10] M. Filali and J. S. Pym, Right cancellation in LUC-compactification of a locally compact group, Bull. London. Math. Soc. 35 (2003), 128-134. 
[11] M. Filali and P. Salmi, One-sided ideals and right cancellation in the second dual of the group algebra and similar algebras, J. London Math. Soc. 75 (2007), $35-46$.

[12] Slowly oscillating functions in semigroup compactifications and convolution algebras, J. Funct. Anal. 250 (2007), 144-166.

[13] _ Topological centres of the weighted convolution algebras, preprint.

[14] M. Filali and A. I. Singh, Recent developments on Arens regularity and ideal structure of the second dual of a group algebra and some related topological algebras, General topological algebras (Tartu, 1999), Math. Stud. (Tartu), vol. 1, Est. Math. Soc., 2001, pp. 95-124.

[15] G. B. Folland, A Course in Abstract Harmonic Analysis, CRC Press, Boca Raton, 1995.

[16] B. Forrest, Arens regularity and discrete groups, Pacific J. Math. 151 (1991), $217-227$.

[17] F. Ghahramani and J. P. McClure, Module homomorphisms of the dual modules of convolution Banach algebras, Canad. Math. Bull. 35 (1992), 180-185.

[18] C. C. Graham, Arens regularity for quotients $A_{p}(E)$ of the Herz algebra, Bull. London Math. Soc. 34 (2002), 457-468.

[19] E. E. Granirer, Day points for quotients of a Fourier algebra $A(G)$, extreme nonergodicity of their duals and extreme non Arens regularity, Illinois J. Math. 40 (1996), 402-419.

[20] E. Hewitt and K. A. Ross, Abstract Harmonic Analysis, Vol. 1, Second edition, Springer-Verlag, Berlin, 1979.

[21] _ Abstract Harmonic Analysis, Vol. 2, Springer-Verlag, New York, 1970.

[22] N. Hindman and D. Strauss, Algebra in the Stone-Cech Compactification, Theory and Applications, Walter de Gruyter, New York, 1998.

[23] H. Hofmeier and G. Wittstock, A bicommutant theorem for completely bounded module homomorphisms, Math. Ann. 308 (1997), 141-154.

[24] Z. Hu, On the set of topologically invariant means on the von Neumann algbera $V N(G)$, Illinois J. Math. 39 (1995), 463-490.

$[25] \_$, Extreme non-Arens regularity of quotients of the Fourier algebra $A(G)$, Colloq. Math. 72 (1997), 237-249.

$[26] \_$Inductive extreme non-Arens regularity of the Fourier algebra $A(G)$, Studia Math. 151 (2002), 247-264.

[27] _ Open subgroups and the centre problem for the Fourier algebra, Proc. Amer. Math. Soc. 134 (2006), 3085-3095.

[28] Z. Hu and M. Neufang, Decomposability of von Nuemann algebras and the Mazur property of higher level, Canad. J. Math. 58 (2006), 768-795.

[29] N. Işik, J. Pym, and A. Ülger, The second dual of the group algebra of a compact group, J. London Math. Soc. 35 (1987), 135-158.

[30] T. Jech, Set Theory, Academic Press, New York, 1978.

[31] E. Kaniuth and A. T.-M. Lau, Fourier algebras and amenability, Banach algebras and their applications, 181-192, Contemp. Math., 363, Amer. Math. Soc., Providence, RI, 2004.

[32] J. Kraus and Z-J. Ruan, Multipliers of Kac algebras, Internat. J. Math. 8 (1997), 213-248.

[33] A. T.-M. Lau, Continuity of Arens multiplication on the dual space of bounded uniformly continuous functions on locally compact groups and topological semigroups, Math. Proc. Cambridge Phil. Soc. 99(1986), 273-283.

[34] A. T.-M. Lau, Uniformly continuous functionals on Banach algebras, Colloquium Math. 51(1987), 195-205. 
[35] A. T.-M. Lau, Fourier and Fourier-Stieltjes algebras of a locally compact group and amenability, Topological vector spaces, algebras and related areas (Hamilton, ON, 1994), 79-92, Pitman Res. Notes Math. Ser., 316, Longman Sci. Tech., Harlow, 1994.

[36] A. T.-M. Lau and V. Losert, On the second conjugate algebra of $L^{1}(G)$ of a locally compact group, J. London Math. Soc. 37 (1988), 464-470.

[37] $\quad$, The $C^{*}$-algbera generated by the operators with compact support on a locally compact group, J. Funct. Anal. 112 (1993), 1-30.

[38] - The centre of the second conjugate algebra of the Fourier algebra for infinite products of groups, Math. Proc. Camb. Phil. Soc. 138 (2005), 27-39.

[39] A. T.-M. Lau and J. C. S. Wong, Weakly almost periodic elements in $L^{\infty}(G)$ of a locally compact group, Proc. Amer. Math. Soc. 107 (1989), 1031-1036.

[40] V. Losert, The centre of the bidual of Fourier algebras (discrete groups), Preprint.

[41] Properties of the Fourier algebra that are equivalent to amenability, Proc. Amer. Math. Soc. 92 (1984), 347-354.

[42] - Further results on the centre of the bidual of Fourier algebras, Lecture given at the Meeting "Harmonic Analysis, Operator Algebras and Representations", CIRM, 03-07/11/2008.

[43] M. Neufang, Solution to a conjecture by Hofmeier-Wittstock, J. Funct. Anal. 217 (2004), 171-180.

[44] _ A unified approach to the topological centre problem for certain Banach algebras arising in abstract harmonic analysis, Arch. Math. 82 (2004), 164171.

[45] _ On a conjecture by Ghahramani-Lau and related problems concerning topological centres, J. Funct. Anal. 224 (2005), 217-229.

[46] A. Yu. Ol'shanskii, An infinite simple Noetherian group without torsion, Math. U.S.S.R.-Izv. 15 (1980), 531-588.

[47] G. Pisier, Multipliers and lacunary sets in non-amenable groups, Amer. J. Math. 117 (1995), 337-376.

[48] G. Pisier, Similarity Problems and Completely Bounded Maps, Second, expanded edition, Lecture Notes in Mathematics, 1618, Springer-Verlag, Berlin, 2001.

[49] E. K. van Douwen, The number of cofinal ultrafilters, Topol. Appl. 39 (1991), 61-63.

[50] N. J. Young, The irregularity of multiplication in group algebras, Quarterly J. Math. Oxford 24 (1973), 59-62.

Department of Mathematical Sciences, University of Oulu, Oulu 90014, FINLAND

E-mail address: mahmoud.filali@oulu.fi

School of Mathematics and Statistics, Carleton University, OtTAWA, ON, K1S 5B6, CANADA.

E-mail address: mneufang@math.carleton.ca

Department of Mathematics and Statistics, University of Windsor, Windsor, ON, N9B 3P4, CANADA.

E-mail address: monfared@uwindsor.ca 